\title{
Certain graphs under Pythagorean fuzzy environment
}

\author{
Muhammad Akram ${ }^{1}$ (D) Jawaria Mohsan $\operatorname{Dar}^{1} \cdot$ Sumera Naz ${ }^{1}$ \\ Received: 29 August 2018 / Accepted: 18 December 2018 / Published online: 3 January 2019 \\ (c) The Author(s) 2019
}

\begin{abstract}
Graph theory plays crucial role in structuring many real-world problems including, medical sciences, control theory, expert systems and network security. Product in graphs, an operation that consider two graphs and produce a new graph by simple or complex changes, has wide range of applications in games theory, automata theory, structural mechanics and networking system. An intuitionistic fuzzy model is used to handle the vagueness and uncertainty in network problems. A Pythagorean fuzzy model is a powerful tool for describing vagueness and uncertainty more accurately as compared to intuitionistic fuzzy model. The objective of this paper is to apply the concept of Pythagorean fuzzy sets to graphs and then combine two Pythagorean fuzzy graphs (PFGs) using two new graph products namely, maximal product and the residue product. This research paper investigates the regularity for these products. Moreover, it discusses some eminent properties such as strongness, connectedness and completeness. Further, it proposes some necessary and sufficient conditions for $\mathscr{G}_{1} * \mathscr{G}_{2}$ and $\mathscr{G}_{1} \cdot \mathscr{G}_{2}$ to be regular. Finally, decision-making problems concerning evaluation of best company for investment and alliance partner selection of a software company are solved to better understand PFGs.
\end{abstract}

Keywords Pythagorean fuzzy graphs $\cdot$ Regular Pythagorean fuzzy graphs $\cdot$ Maximal product $\cdot$ Residue product

\section{Introduction}

Fuzzy set theory [1] is the most efficient tool having the capability to deal with imprecise and incomplete information in different disciplines, including engineering, mathematics, statistics, artificial intelligence, medical and social sciences. To cope with imprecise and incomplete information, consisting of doubts in human judgement, the fuzzy set shows some restrictions. So, for characterizing the hesitancy more explicitly, fuzzy sets were extended to intuitionistic fuzzy sets (IFSs) by Atanassov [2], which assign a membership grade $(\mu)$ and a non-membership grade $(v)$ to the objects, satisfying the condition $\mu+v \leq 1$ and the hesitancy part $\pi=1-\mu-v$. The IFSs have gained extensive attentions and have been broadly applied in different areas of real life. The limitation $\mu+v \leq 1$ confines the choice of the mem-

Muhammad Akram

m.akram@pucit.edu.pk

Jawaria Mohsan Dar

jiadar4@gmail.com

Sumera Naz

nsumeranaz@gmail.com

1 Department of Mathematics, University of the Punjab, New Campus, Lahore 4590, Pakistan bership and non-membership grades in IFS. To evade this situation, Yager [3-5] initiated the idea of Pythagorean fuzzy set (PFS), depicted by a membership grade $(\mu)$ and a nonmembership grade $(v)$ with the condition $\mu^{2}+v^{2} \leq 1$. Zhang and $\mathrm{Xu}[6]$ introduced the concept of Pythagorean fuzzy number (PFN) for interpreting the dual aspects of an element. In a decision-making environment, a specialist gives the preference information about an alternative with the membership grade 0.9 and the non-membership grade 0.3 ; it is noted that the IFN fails to address this situation, as $0.9+0.3>$ 1 , but $(0.9)^{2}+(0.3)^{2} \leq 1$. Thus, the PFS has much stronger ability than IFS to model fuzziness in the practical MCDM problems. Under Pythagorean fuzzy environment, many researchers have initiated work in different directions and acquired various eminent results [7]. Some operations on PFSs [8] and Pythagorean fuzzy TODIM approach to multi-criteria decision-making [9] have been discussed. Furthermore, the PFS has been investigated from different perspectives, including aggregation operators [10,11]. Garg [12-18] explored applications of Pythagorean fuzzy sets in decision-making. Lately, the concept of Pythagorean fuzzy set has been extended to interval-valued Pythagorean fuzzy set and hesitant Pythagorean fuzzy set. Garg [19,20] elaborated exponential operational laws and their aggregation operators under interval-valued Pythagorean fuzzy informa-

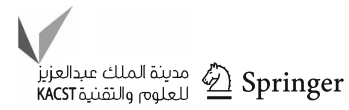


tion. Yu et al. [21] discussed hesitant Pythagorean fuzzy Maclaurin symmetric mean operators and its applications to multi-attribute decision-making.

Graphs are the pictorial representation that bond the objects and highlight their information. Graph theory is rapidly moving into the core of mathematics due to its applications in various fields, including physics, biochemistry, biology, electrical engineering, astronomy, operations research and computer science. To emphasize on a real-world problem, the bondedness between the objects occur due to some relations. But when there exists uncertainty and haziness in the bonding, then the corresponding graph model can be taken as fuzzy graph model. For example, a social network may be represented as a graph, where vertices represent members and edges represent relation between members. If the relations among the members are to be measured as good or bad, then fuzziness should be added to the representation. This and many other problems motivated to define fuzzy graph. The concept of fuzzy graphs was presented by Kaufmann [22], based on Zadeh's fuzzy relation in 1971. Later, Rosenfeld [23] discussed several basic graph theoretical concepts, including paths, cycles, bridges and connectedness in fuzzy environment. Nagoor Gani and Radha [24] initiated the concept of regular fuzzy graph in 2008. Mordeson and Peng [25] introduced some operations on fuzzy graphs and studied their properties. Further, Nirmala and Vijaya [26] explored new operations on fuzzy graphs. Parvathi and Karunambigai [27] considered intuitionistic fuzzy graphs (IFGs). Later, Akram and Davvaz [28] discussed IFGs. An algorithm for computing sum distance matrix, eccentricity of vertices, diameter and radius in IFGs was presented by sarwar and Akram [29]. Akram and Dudek [30] described intuitionistic fuzzy hypergraphs with applications. Recently, Naz et al. [31] originally proposed the concept of PFGs, a generalization of the notion of Akram and Davvaz's IFGs [28], along with its applications in decision-making. Akram and Naz [32] studied energy of PFGs with applications. Akram et al. [33] introduced the concept of Pythagorean fuzzy Planar graphs. Dhavudh and Srinivasan [35] dealt with IFGs2T. Ghorai and Pal [37,38] studied some properties of $m$-polar fuzzy graphs. Recently, Akram et al. [34] introduced some new operations including rejection, symmetric difference, maximal product and residue product of PFGs. This paper describes two new operations namely, maximal product and residue product of PFGs that can allow the mathematical design of network. These products can be applied to construct and analyze several real-world networks such as road networks and communication networks. The work explores some significant properties such as regularity, strongness, completeness and connectedness. As regularity plays a substantial role in designing reliable communication networks, so the main focus is to familiarize the regularity of these product with illustrative examples. The work also proposes some necessary and sufficient conditions for these two products to be regular. Finally, it discusses some applications of PFGs in decision-making.

For better understanding, we present prerequisite terminologies and notations:

Definition 1.1 [1] A fuzzy set (FS) on a universe $\mathscr{X}$ is an object of the form

$\mathscr{A}=\left\{\left\langle u, \mu_{\mathscr{A}}(u)\right\rangle \mid u \in \mathscr{X}\right\}$,

where $\mu_{\mathscr{A}}: \mathscr{X} \longrightarrow[0,1]$ represents the membership functions of $\mathscr{A}$.

Definition 1.2 [22] A fuzzy graph (FG) on a non-empty set $\mathscr{X}$ is a pair $\mathscr{G}=(\mathscr{A}, \mathscr{B})$ with $\mathscr{A}$ a FS on $\mathscr{X}$ and $\mathscr{B}$ a fuzzy relation on $\mathscr{X}$ such that

$\mu_{\mathscr{B}}(u v) \leq \mu_{\mathscr{A}}(u) \wedge \mu_{\mathscr{A}}(v)$

for all $u, v \in \mathscr{X}$, where $\mathscr{A}: \mathscr{X} \longrightarrow[0,1]$ and $\mathscr{B}:$ $\mathscr{X} \times \mathscr{X} \longrightarrow[0,1]$.

Definition 1.3 [2] An intuitionistic fuzzy set (IFS) on a universe $\mathscr{X}$ is an object of the form

$\mathscr{A}=\left\{\left\langle u, \mu_{\mathscr{A}}(u), v_{\mathscr{A}}(u)\right\rangle \mid u \in \mathscr{X}\right\}$,

where $\mu_{\mathscr{A}}: \mathscr{X} \longrightarrow[0,1]$ and $v_{\mathscr{A}}: \mathscr{X} \longrightarrow[0,1]$ represents the membership and non-membership functions of $\mathscr{A}$, and $\mu_{\mathscr{A}}, v_{\mathscr{A}}$ satisfies the condition $0 \leq \mu_{\mathscr{A}}(u)+v_{\mathscr{A}}(u) \leq 1$ for all $u \in \mathscr{X}$.

Definition 1.4 [27] An intuitionistic fuzzy graph (IFG) on a non-empty set $\mathscr{X}$ is a pair $\mathscr{G}=(\mathscr{A}, \mathscr{B})$ with $\mathscr{A}$ an IFS on $\mathscr{X}$ and $\mathscr{B}$ an IFR on $\mathscr{X}$ such that

$\mu_{\mathscr{B}}(u v) \leq \mu_{\mathscr{A}}(u) \wedge \mu_{\mathscr{A}}(v), v_{\mathscr{B}}(u v) \geq v_{\mathscr{A}}(u) \vee v_{\mathscr{A}}(v)$

and $0 \leq \mu_{\mathscr{B}}(u v)+v_{\mathscr{B}}(u v) \leq 1$ for all $u, v \in \mathscr{X}$, where, $\mu_{\mathscr{B}}: \mathscr{X} \times \mathscr{X} \longrightarrow[0,1]$ and $\nu_{\mathscr{B}}: \mathscr{X} \times \mathscr{X} \longrightarrow[0,1]$ represents the membership and non-membership functions of $\mathscr{B}$, respectively.

Definition 1.5 [3] A Pythagorean fuzzy set (PFS) on a universe $\mathscr{X}$ is an object of the form

$\mathscr{A}=\left\{\left\langle u, \mu_{\mathscr{A}}(u), v_{\mathscr{A}}(u)\right\rangle \mid u \in \mathscr{X}\right\}$,

where $\mu_{\mathscr{A}}: \mathscr{X} \longrightarrow[0,1]$ and $v_{\mathscr{A}}: \mathscr{X} \longrightarrow[0,1]$ represents the membership and non-membership functions of $\mathscr{A}$, and $\mu_{\mathscr{A}}, v_{\mathscr{A}}$ satisfies the condition $0 \leq \mu_{\mathscr{A}}^{2}(u)+v_{\mathscr{A}}^{2}(u) \leq 1$ for all $u \in \mathscr{X}$. 
Fig. 1 Pythagorean fuzzy graph

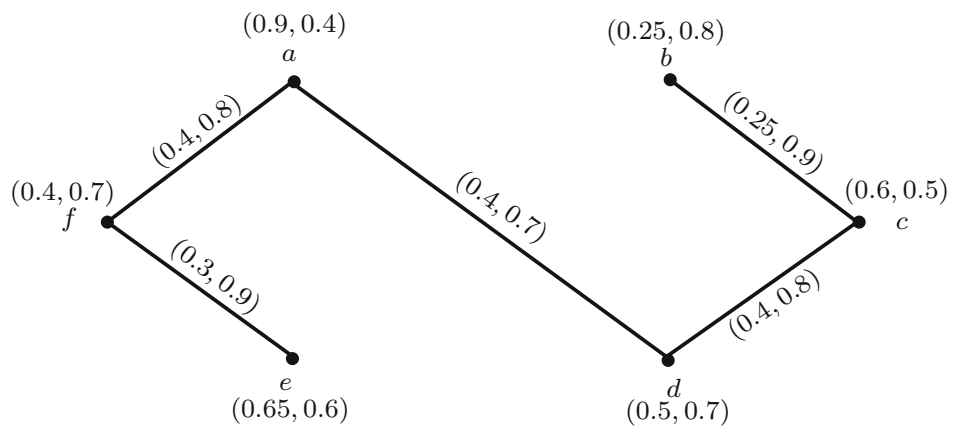

Definition 1.6 Let $\mathscr{A}_{1}=\left\{\left\langle u, \mu_{\mathscr{A}_{1}}(u), v_{\mathscr{A}_{1}}(u)\right\rangle \mid u \in \mathscr{X}\right\}$ and $\mathscr{A}_{2}=\left\{\left\langle u, \mu_{\mathscr{A}_{2}}(u), v_{\mathscr{A}_{2}}(u)\right\rangle \mid u \in \mathscr{X}\right\}$ be two Pythagorean fuzzy sets, then some set operations can be defined as

1. $\mathscr{A}_{1} \subseteq \mathscr{A}_{2}$ if and only if $\mu_{\mathscr{A}_{1}}(u) \leq \mu_{\mathscr{A}_{2}}(u)$ and $v_{\mathscr{A}_{1}}(u) \geq$ $v_{\mathscr{A}_{2}}(u)$,

2. $\mathscr{A}_{1}=\mathscr{A}_{2}$ if and only if $\mathscr{A}_{1}(u) \subseteq \mathscr{A}_{2}(u)$ and $\mathscr{A}_{2}(u) \subseteq$ $\mathscr{A}_{1}(u)$

3. $\mathscr{A}_{1}^{c}=\left\{\left\langle u, v_{\mathscr{A}_{1}}(u), \mu_{\mathscr{A}_{1}}(u)\right\rangle \mid u \in \mathscr{X}\right\}$,

4. $\mathscr{A}_{1} \cap \mathscr{A}_{2}=\left\{\left\langle u, \min \left\{\mu_{\mathscr{A}_{1}}(u), \mu_{\mathscr{A}_{2}}(u)\right\}, \max \left\{v_{\mathscr{A}_{1}}(u)\right.\right.\right.$, $\left.\left.\left.v_{\mathscr{A}_{2}}(u)\right\}\right\rangle \mid u \in \mathscr{X}\right\}$,

5. $\mathscr{A}_{1} \cup \mathscr{A}_{2}=\left\{\left\langle u, \max \left\{\mu_{\mathscr{A}_{1}}(u), \mu_{\mathscr{A}_{2}}(u)\right\}, \min \left\{v_{\mathscr{A}_{1}}(u)\right.\right.\right.$, $\left.\left.\left.v_{\mathscr{A}_{2}}(u)\right\}\right\rangle \mid u \in \mathscr{X}\right\}$.

Definition 1.7 [31] A Pythagorean fuzzy set $\mathscr{B}$ on $\mathscr{X} \times \mathscr{X}$ is said to be a Pythagorean fuzzy relation (PFR) on $\mathscr{X}$, denoted by

$\mathscr{B}=\left\{\left\langle u v, \mu_{\mathscr{B}}(u v), v_{\mathscr{B}}(u v)\right\rangle \mid u v \in \mathscr{X} \times \mathscr{X}\right\}$,

where $\mu_{\mathscr{B}}: \mathscr{X} \times \mathscr{X} \longrightarrow[0,1]$ and $\nu_{\mathscr{B}}: \mathscr{X} \times \mathscr{X} \longrightarrow$ $[0,1]$ represents the membership and non-membership functions of $\mathscr{B}$, and $\mu_{\mathscr{B}}, v_{\mathscr{B}}$ satisfies the condition $0 \leq$ $\mu_{\mathscr{B}}^{2}(u v)+v_{\mathscr{B}}^{2}(u v) \leq 1$ for all $u v \in \mathscr{X} \times \mathscr{X}$.

Definition 1.8 [31] A Pythagorean fuzzy graph (PFG) on a non-empty set $\mathscr{X}$ is a pair $\mathscr{G}=(\mathscr{A}, \mathscr{B})$ with $\mathscr{A}$ a PFS on $\mathscr{X}$ and $\mathscr{B}$ a PFR on $\mathscr{X}$ such that

$\mu_{\mathscr{B}}(u v) \leq \mu_{\mathscr{A}}(u) \wedge \mu_{\mathscr{A}}(v), v_{\mathscr{B}}(u v) \geq v_{\mathscr{A}}(u) \vee v_{\mathscr{A}}(v)$

and $0 \leq \mu_{\mathscr{B}}^{2}(u v)+v_{\mathscr{B}}^{2}(u v) \leq 1$ for all $u, v \in \mathscr{X}$, where, $\mu_{\mathscr{B}}: \mathscr{X} \times \mathscr{X} \longrightarrow[0,1]$ and $\nu_{\mathscr{B}}: \mathscr{X} \times \mathscr{X} \longrightarrow[0,1]$ represents the membership and non-membership functions of $\mathscr{B}$, respectively.

Example 1.1 Consider a graph $G=(V, E)$ where $V=$ $\{a, b, c, d, e, f\}$ and $E=\{a d, b c, c d, a f, e f\}$. Let $\mathscr{A}$ and $\mathscr{B}$ be Pythagorean fuzzy vertex set and Pythagorean fuzzy edge set defined on $V$ and $V \times V$, respectively.

$$
\begin{aligned}
\mathscr{A}= & \left\langle\left(\frac{a}{0.9}, \frac{b}{0.25}, \frac{c}{0.6}, \frac{d}{0.5}, \frac{e}{0.65}, \frac{f}{0.4}\right),\right. \\
& \left.\times\left(\frac{a}{0.4}, \frac{b}{0.8}, \frac{c}{0.5}, \frac{d}{0.7}, \frac{e}{0.6} \frac{f}{0.7}\right)\right\rangle \text { and } \\
\mathscr{B}= & \left\langle\left(\frac{a d}{0.4}, \frac{b c}{0.25}, \frac{c d}{0.4}, \frac{a f}{0.4}, \frac{f e}{0.3}\right),\right. \\
& \left.\times\left(\frac{a d}{0.7}, \frac{b c}{0.9}, \frac{c d}{0.8}, \frac{a f}{0.8}, \frac{f e}{0.9}\right)\right\rangle .
\end{aligned}
$$

By routine calculation, one can see from Fig. 1 that it is a Pythagorean fuzzy graph.

Definition 1.9 A Pythagorean fuzzy preference relation (PFPR) on the set of alternatives $\mathscr{X}=\left\{x_{1}, x_{2}, \ldots, x_{n}\right\}$ is represented by a matrix $R=\left(r_{i j}\right)_{n \times n}$, where $r_{i j}=$ $\left(x_{i} x_{j}, \mu\left(x_{i} x_{j}\right), v\left(x_{i} x_{j}\right)\right)$ for all $i, j=1,2, \ldots, n$. For convenience, let $r_{i j}=\left(\mu_{i j}, v_{i j}\right)$ where $\mu_{i j}$ indicates the degree to which the object $x_{i}$ is preferred to the object $x_{j}, v_{i j}$ denotes the degree to which the object $x_{i}$ is not preferred to the object $x_{j}$, and $\pi_{i j}=\sqrt{1-\mu_{i j}^{2}-v_{i j}^{2}}$ is interpreted as a hesitancy degree, with the conditions:

$$
\begin{gathered}
\mu_{i j}, v_{i j} \in[0,1], \mu_{i j}^{2}+v_{i j}^{2} \leq 1, \mu_{i j}=v_{j i}, \\
\mu_{i i}=v_{i i}=0.5, \text { for all } i, j=1,2, \ldots, n .
\end{gathered}
$$

\section{Regular maximal product in Pythagorean fuzzy graphs}

Definition 2.1 Let $\mathscr{G}_{1}=\left(\mathscr{A}_{1}, \mathscr{B}_{1}\right)$ and $\mathscr{G}_{2}=\left(\mathscr{A}_{2}, \mathscr{B}_{2}\right)$ be two PFGs of $G_{1}=\left(V_{1}, E_{1}\right)$ and $G_{2}=\left(V_{2}, E_{2}\right)$, respectively. The maximal product of $\mathscr{G}_{1}$ and $\mathscr{G}_{2}$ is denoted by $\mathscr{G}_{1} * \mathscr{G}_{2}=\left(\mathscr{A}_{1} * \mathscr{A}_{2}, \mathscr{B}_{1} * \mathscr{B}_{2}\right)$ and defined as:

(i) $\left\{\begin{array}{l}\left(\mu_{\mathscr{A}_{1}} * \mu_{\mathscr{A}_{2}}\right)\left(u_{1}, u_{2}\right)=\mu_{\mathscr{A}_{1}}\left(u_{1}\right) \vee \mu_{\mathscr{L}_{2}}\left(u_{2}\right) \\ \left(v_{\mathscr{A}_{1}} * v_{\mathscr{A}_{2}}\right)\left(u_{1}, u_{2}\right)=v_{\mathscr{A}_{1}}\left(u_{1}\right) \wedge v_{\mathscr{L}_{2}}\left(u_{2}\right)\end{array}\right.$

for all $\left(u_{1}, u_{2}\right) \in V_{1} \times V_{2}$,

(ii) $\left\{\begin{array}{l}\left(\mu_{\mathscr{B}_{1}} * \mu_{\mathscr{B}_{2}}\right)\left(\left(u, u_{2}\right)\left(u, v_{2}\right)\right)=\mu_{\mathscr{A}_{1}}(u) \vee \mu_{\mathscr{B}_{2}}\left(u_{2} v_{2}\right) \\ \left(v_{\mathscr{B}_{1}} * v_{\mathscr{B}_{2}}\right)\left(\left(u, u_{2}\right)\left(u, v_{2}\right)\right)=v_{\mathscr{A}_{1}}(u) \wedge v_{\mathscr{B}_{2}}\left(u_{2} v_{2}\right)\end{array}\right.$ for all $u \in V_{1}$ and $u_{2} v_{2} \in E_{2}$, 


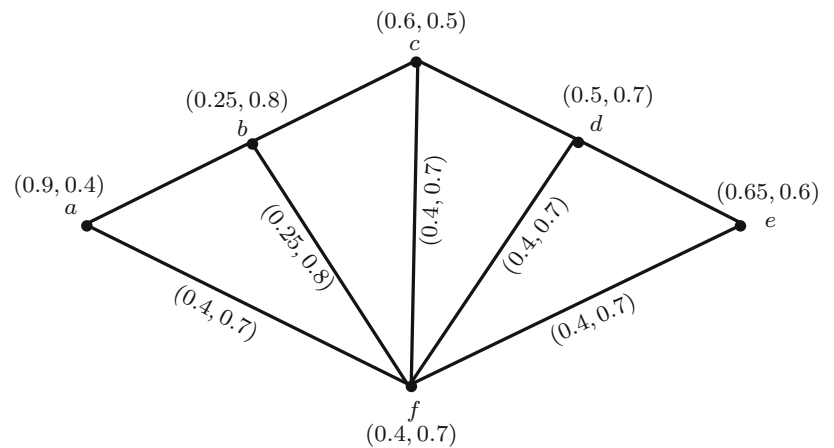

Fig. 2 Strong Pythagorean fuzzy graph

(iii) $\left\{\begin{array}{l}\left(\mu_{\mathscr{B}_{1}} * \mu_{\mathscr{B}_{2}}\right)\left(\left(u_{1}, z\right)\left(v_{1}, z\right)\right)=\mu_{\mathscr{B}_{1}}\left(u_{1} v_{1}\right) \vee \mu_{\mathscr{A}_{2}}(z) \\ \left(v_{\mathscr{B}_{1}} * v_{\mathscr{B}_{2}}\right)\left(\left(u_{1}, z\right)\left(v_{1}, z\right)\right)=v_{\mathscr{B}_{1}}\left(u_{1} v_{1}\right) \wedge v_{\mathscr{A}_{2}}(z)\end{array}\right.$ for all $z \in V_{2}$ and $u_{1} v_{1} \in E_{1}$.

Definition 2.2 A Pythagorean fuzzy graph $\mathscr{G}=(\mathscr{A}, \mathscr{B})$ is said to be a strong Pythagorean fuzzy graph of underlying crisp graph $G=(V, E)$ if

$\mu_{\mathscr{B}}(u v)=\mu_{\mathscr{A}}(u) \wedge \mu_{\mathscr{A}}(v)$,

$v_{\mathscr{B}}(u v)=v_{\mathscr{A}}(u) \vee v_{\mathscr{A}}(v)$ for all uv $\in \mathrm{E}$.

Example 2.1 Consider a graph $G=(V, E)$ where $V=$ $\{a, b, c, d, e, f\}$ and $E=\{a f, b f, c f, d f, e f\}$. Let $\mathscr{A}$ and $\mathscr{B}$ be Pythagorean fuzzy vertex set and Pythagorean fuzzy edge set defined on $V$ and $V \times V$, respectively.

$$
\begin{aligned}
\mathscr{A}= & \left\langle\left(\frac{a}{0.9}, \frac{b}{0.25}, \frac{c}{0.6}, \frac{d}{0.5}, \frac{e}{0.65}, \frac{f}{0.4}\right),\right. \\
& \left.\times\left(\frac{a}{0.4}, \frac{b}{0.8}, \frac{c}{0.5}, \frac{d}{0.7}, \frac{e}{0.6}, \frac{f}{0.7}\right)\right\rangle \text { and } \\
\mathscr{B}= & \left\langle\left(\frac{a f}{0.4}, \frac{b f}{0.25}, \frac{c f}{0.4}, \frac{d f}{0.4}, \frac{e f}{0.4}\right),\right. \\
& \left.\times\left(\frac{a f}{0.7}, \frac{b f}{0.8}, \frac{c f}{0.7}, \frac{d f}{0.7}, \frac{e f}{0.7}\right)\right\rangle .
\end{aligned}
$$

By routine calculation, one can see from Fig. 2 that it is a strong Pythagorean fuzzy graph.

Theorem 2.1 If $\mathscr{G}_{1}=\left(\mathscr{A}_{1}, \mathscr{B}_{1}\right)$ and $\mathscr{G}_{2}=\left(\mathscr{A}_{2}, \mathscr{B}_{2}\right)$ are two strong PFGs, then their maximal product is also a strong $P F G$.

Proof Let $\mathscr{G}_{1}=\left(\mathscr{A}_{1}, \mathscr{B}_{1}\right)$ and $\mathscr{G}_{2}=\left(\mathscr{A}_{2}, \mathscr{B}_{2}\right)$ be two strong PFGs of the graph $G_{1}$ and $G_{2}$, respectively. Then,

$$
\begin{aligned}
& \mu_{\mathscr{B}_{1}}\left(u_{1} u_{2}\right)=\mu_{\mathscr{A}_{1}}\left(u_{1}\right) \wedge \mu_{\mathscr{A}_{1}}\left(u_{2}\right), \\
& v_{\mathscr{B}_{1}}\left(u_{1} u_{2}\right)=v_{\mathscr{A}_{1}}\left(u_{1}\right) \vee v_{\mathscr{A}_{1}}\left(u_{2}\right) \text { for all } u_{1} u_{2} \in E_{1}, \\
& \mu_{\mathscr{B}_{2}}\left(v_{1} v_{2}\right)=\mu_{\mathscr{A}_{2}}\left(v_{1}\right) \wedge \mu_{\mathscr{A}_{2}}\left(v_{2}\right), \\
& v_{\mathscr{B}_{2}}\left(v_{1} v_{2}\right)=v_{\mathscr{A}_{2}}\left(v_{1}\right) \vee v_{\mathscr{A}_{2}}\left(v_{2}\right) \text { for all } v_{1} v_{2} \in E_{2} .
\end{aligned}
$$

By definition of maximal product, we have

$$
\begin{aligned}
& \text { If } u_{1}=u_{2} \text { and } v_{1} v_{2} \in E_{2}, \\
& \begin{aligned}
\left(\mu_{\mathscr{B}_{1}} * \mu_{\mathscr{B}_{2}}\right)\left(\left(u_{1}, v_{1}\right)\left(u_{2}, v_{2}\right)\right) \\
\quad=\mu_{\mathscr{A}_{1}}\left(u_{1}\right) \vee \mu_{\mathscr{B}_{2}}\left(v_{1} v_{2}\right) \\
\quad=\mu_{\mathscr{A}_{1}}\left(u_{1}\right) \vee\left\{\mu_{\mathscr{A}_{2}}\left(v_{1}\right) \wedge \mu_{\mathscr{A}_{2}}\left(v_{2}\right)\right\} \\
\quad=\left\{\mu_{\mathscr{A}_{1}}\left(u_{1}\right) \vee \mu_{\mathscr{A}_{2}}\left(v_{1}\right)\right\} \wedge\left\{\mu_{\mathscr{A}_{1}}\left(u_{1}\right) \vee \mu_{\mathscr{A}_{2}}\left(v_{2}\right)\right\} \\
\quad=\left(\mu_{\mathscr{A}_{1}} * \mu_{\mathscr{A}_{2}}\right)\left(u_{1}, v_{1}\right) \wedge\left(\mu_{\mathscr{A}_{1}} * \mu_{\mathscr{A}_{2}}\right)\left(u_{2}, v_{2}\right), \\
\left(v_{\mathscr{B}_{1}} * v_{\mathscr{B}_{2}}\right)\left(\left(u_{1}, v_{1}\right)\left(u_{2}, v_{2}\right)\right) \\
\quad=v_{\mathscr{A}_{1}}\left(u_{1}\right) \wedge v_{\mathscr{B}_{2}}\left(v_{1} v_{2}\right) \\
=v_{\mathscr{A}_{1}}\left(u_{1}\right) \wedge\left\{v_{\mathscr{A}_{2}}\left(v_{1}\right) \vee v_{\mathscr{A}_{2}}\left(v_{2}\right)\right\} \\
=\left\{v_{\mathscr{A}_{1}}\left(u_{1}\right) \wedge v_{\mathscr{A}_{2}}\left(v_{1}\right)\right\} \vee\left\{v_{\mathscr{A}_{1}}\left(u_{1}\right) \wedge v_{\mathscr{A}_{2}}\left(v_{2}\right)\right\} \\
=\left(v_{\mathscr{A}_{1}} * v_{\mathscr{A}_{2}}\right)\left(u_{1}, v_{1}\right) \vee\left(v_{\mathscr{A}_{1}} * v_{\mathscr{A}_{2}}\right)\left(u_{2}, v_{2}\right) .
\end{aligned}
\end{aligned}
$$

If $v_{1}=v_{2}$ and $u_{1} u_{2} \in E_{1}$,

$$
\begin{aligned}
& \left(\mu_{\mathscr{B}_{1}} * \mu_{\mathscr{B}_{2}}\right)\left(\left(u_{1}, v_{1}\right)\left(u_{2}, v_{2}\right)\right) \\
& \quad=\mu \mathscr{B}_{1}\left(u_{1} u_{2}\right) \vee \mu_{\mathscr{A}_{2}}\left(v_{1}\right) \\
& \quad=\left\{\mu_{\mathscr{A}_{1}}\left(u_{1}\right) \wedge \mu_{\mathscr{A}_{1}}\left(u_{2}\right)\right\} \vee \mu_{\mathscr{A}_{2}}\left(v_{1}\right) \\
& \quad=\left\{\mu_{\mathscr{A}_{1}}\left(u_{1}\right) \vee \mu_{\mathscr{A}_{2}}\left(v_{1}\right)\right\} \wedge\left\{\mu_{\mathscr{A}_{1}}\left(u_{2}\right) \vee \mu_{\mathscr{A}_{2}}\left(v_{1}\right)\right\} \\
& \quad=\left(\mu_{\mathscr{A}_{1}} * \mu_{\mathscr{A}_{2}}\right)\left(u_{1}, v_{1}\right) \wedge\left(\mu_{\mathscr{A}_{1}} * \mu_{\mathscr{A}_{2}}\right)\left(u_{2}, v_{2}\right), \\
& \left(v_{\mathscr{B}_{1}} * v_{\mathscr{B}_{2}}\right)\left(\left(u_{1}, v_{1}\right)\left(u_{2}, v_{2}\right)\right) \\
& \quad=v_{\mathscr{B}_{1}}\left(u_{1} u_{2}\right) \wedge v_{\mathscr{A}_{2}}\left(v_{1}\right) \\
& \quad=\left\{v_{\mathscr{A}_{1}}\left(u_{1}\right) \vee v_{\mathscr{A}_{1}}\left(u_{2}\right)\right\} \wedge v_{\mathscr{A}_{2}}\left(v_{1}\right) \\
& \quad=\left\{v_{\mathscr{A}_{1}}\left(u_{1}\right) \wedge v_{\mathscr{A}_{2}}\left(v_{1}\right)\right\} \vee\left\{v_{\mathscr{A}_{1}}\left(u_{2}\right) \wedge v_{\mathscr{A}_{2}}\left(v_{1}\right)\right\} \\
& \quad=\left(v_{\mathscr{A}_{1}} * v_{\mathscr{A}_{2}}\right)\left(u_{1}, v_{1}\right) \vee\left(v_{\mathscr{A}_{1}} * v_{\mathscr{A}_{2}}\right)\left(u_{2}, v_{2}\right) .
\end{aligned}
$$

Hence, the maximal product $\mathscr{G}_{1} * \mathscr{G}_{2}$ of two strong PFGs is a strong PFG.

Remark 2.1 Converse of the Theorem 2.1 may not be true as it can be seen in the following example.

Consider two Pythagorean fuzzy graphs $\mathscr{G}_{1}=\left(\mathscr{A}_{1}, \mathscr{B}_{1}\right)$ and $\mathscr{G}_{2}=\left(\mathscr{A}_{2}, \mathscr{B}_{2}\right)$ as shown in Fig. 3 , their maximal product is given in Fig. 4.

Here $\mu_{\mathscr{B}_{1}}\left(u_{1} u_{2}\right) \neq \mu_{\mathscr{A}_{1}}\left(u_{1}\right) \wedge \mu_{\mathscr{A}_{1}}\left(u_{2}\right), v_{\mathscr{B}_{1}}\left(u_{1} u_{2}\right) \neq$ $v_{\mathscr{A}_{1}}\left(u_{1}\right) \vee v_{\mathscr{A}_{1}}\left(u_{2}\right)$ and $\mu_{\mathscr{B}_{2}}\left(v_{1} v_{2}\right) \neq \mu_{\mathscr{A}_{2}}\left(v_{1}\right) \wedge \mu_{\mathscr{A}_{2}}\left(v_{2}\right)$, $v_{\mathscr{B}_{2}}\left(v_{1} v_{2}\right) \neq v_{\mathscr{A}_{2}}\left(v_{1}\right) \vee v_{\mathscr{A}_{2}}\left(v_{2}\right)$. Hence, $\mathscr{G}_{1}$ and $\mathscr{G}_{2}$ are not strong PFGs.

But $\left(\mu_{\mathscr{B}_{1}} * \mu_{\mathscr{B}_{2}}\right)((u, v)(x, y))=\mu_{\mathscr{A}_{1}}(u, v) \wedge \mu_{\mathscr{A}_{2}}(x, y)$, $\left(v_{\mathscr{B}_{1}} * \mu_{\mathscr{B}_{2}}\right)((u, v)(x, y))=v_{\mathscr{A}_{1}}(u, v) \vee v_{\mathscr{A}_{2}}(x, y)$ for all edge $(u, v)(x, y)$ in E. Thus, their maximal product $\mathscr{G}_{1} * \mathscr{G}_{2}$ is a strong PFG.

Definition 2.3 Let $\mathscr{G}=(\mathscr{A}, \mathscr{B})$ be a Pythagorean fuzzy graph on underlying crisp graph $G=(V, E) . \mathcal{G}$ is said to be connected, if for every pair of vertices, there exist at least one non-zero path, that is, for all $u, v \in V$, the $\mu$-strength of connectedness 


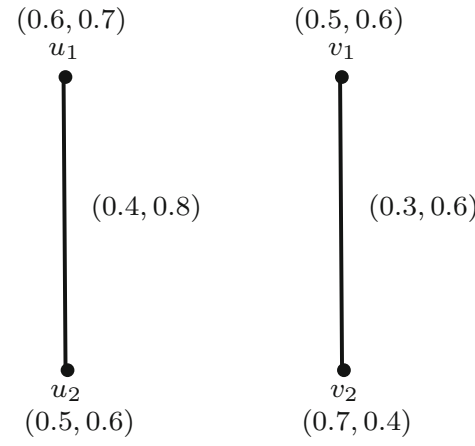

(a) $\mathscr{G}_{1}$

Fig. 3 Pythagorean fuzzy graphs

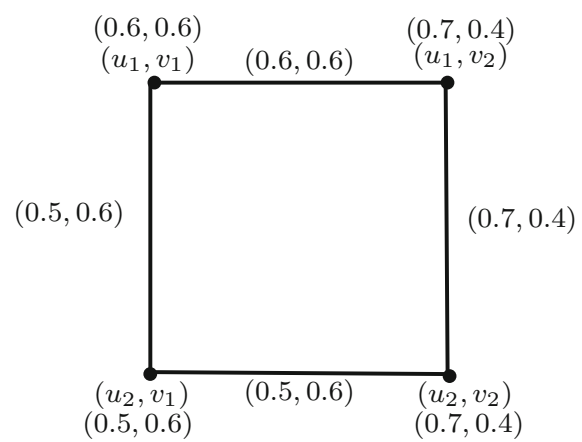

Fig. 4 Maximal product of PFGs

$\mu^{\infty}(u, v)=\sup \left\{\mu^{l}(u, v):\right.$ for some 1$\}$

and $v$-strength of connectedness

$v^{\infty}(u, v)=\inf \left\{v^{l}(u, v):\right.$ for some 1$\}$

satisfy one of the following conditions:

$$
\begin{aligned}
& \mu^{\infty}(u, v)>0 \text { and } v^{\infty}(u, v)>0 \\
& \quad \text { or } \mu^{\infty}(u, v)=0 \text { and } v^{\infty}(u, v)>0 \\
& \quad \text { or } \mu^{\infty}(u, v)>0 \text { and } v^{\infty}(u, v)=0 .
\end{aligned}
$$

where,

$\mu^{l}(u, v)=\mu\left(u u_{1}\right) \wedge \mu\left(u_{1} u_{2}\right) \wedge \ldots \wedge \mu\left(u_{l-1} v\right)$,

$v^{l}(u, v)=v\left(u, u_{1}\right) \vee v\left(u_{1} u_{2}\right) \vee \ldots \vee v\left(u_{l-1} v\right)$ for some 1

represents $\mu$-strength of path and $\nu$-strength of path of length $l$, respectively.

Example 2.2 Consider a Pythagorean fuzzy graph $\mathscr{G}=$ $(\mathscr{A}, \mathscr{B})$ as displayed in the following Fig. 5.

For $b, f \in V$, using Definition 2.3 we have

$$
\begin{aligned}
\mu^{5}(b, f)= & \mu_{\mathscr{B}}(b, c) \wedge \mu_{\mathscr{B}}(c, i) \wedge \mu_{\mathscr{B}}(i, h) \wedge \mu_{\mathscr{B}}(h, g) \\
& \wedge \mu_{\mathscr{B}}(g, f)=0.6 \wedge 0.2 \wedge 0.4
\end{aligned}
$$

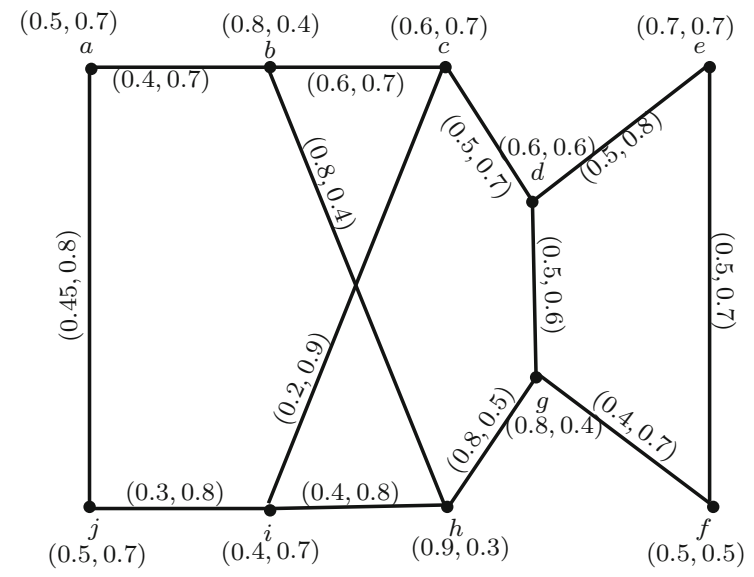

Fig. $5 \mathscr{G}=(\mathscr{A}, \mathscr{B})$

$$
\begin{aligned}
& \wedge 0.8 \wedge 0.4=0.2, \\
\mu^{4}(b, f)= & \mu_{\mathscr{B}}(b, c) \wedge \mu_{\mathscr{B}}(c, d) \wedge \mu_{\mathscr{B}}(d, e) \wedge \mu_{\mathscr{B}}(e, f) \\
= & 0.6 \wedge 0.5 \wedge 0.5 \wedge 0.5=0.5, \\
\mu^{4}(b, f)= & \mu_{\mathscr{B}}(b, c) \wedge \mu_{\mathscr{B}}(c, d) \wedge \mu_{\mathscr{B}}(d, g) \wedge \mu_{\mathscr{B}}(g, f) \\
= & 0.6 \wedge 0.5 \wedge 0.5 \wedge 0.4=0.4, \\
\mu^{3}(b, f)= & \mu_{\mathscr{B}}(b, h) \wedge \mu_{\mathscr{B}}(h, g) \wedge \mu_{\mathscr{B}}(g, f) \\
= & 0.8 \wedge 0.8 \wedge 0.4=0.4 .
\end{aligned}
$$

This implies

$$
\begin{aligned}
\mu^{\infty}(b, f) & =\sup \left\{\mu^{5}(b, f), \mu^{4}(b, f), \mu^{4}(b, f), \mu^{3}(b, f)\right\} \\
& =\sup \{0.2,0.5,0.4,0.4\}=0.5>0,
\end{aligned}
$$

and

$$
\begin{aligned}
v^{5}(b, f)= & v_{\mathscr{B}}(b, c) \vee v_{\mathscr{B}}(c, i) \vee v_{\mathscr{B}}(i, h) \vee v_{\mathscr{B}}(h, g) \\
& \vee v_{\mathscr{B}}(g, f)=0.7 \vee 0.9 \vee 0.8 \vee 0.5 \vee 0.7=0.9, \\
v^{4}(b, f)= & v_{\mathscr{B}}(b, c) \vee v_{\mathscr{B}}(c, d) \vee v_{\mathscr{B}}(d, e) \vee v_{\mathscr{B}}(e, f) \\
= & 0.4 \vee 0.7 \vee 0.8 \vee 0.7=0.8, \\
v^{4}(b, f)= & \mu_{\mathscr{B}}(b, c) \vee v_{\mathscr{B}}(c, d) \vee v_{\mathscr{B}}(d, g) \vee v_{\mathscr{B}}(g, f) \\
= & 0.4 \vee 0.7 \vee 0.6 \vee 0.7=0.7, \\
v^{3}(b, f)= & v_{\mathscr{B}}(b, h) \vee v_{\mathscr{B}}(h, g) \vee v_{\mathscr{B}}(g, f) \\
= & 0.4 \vee 0.5 \vee 0.7=0.7 .
\end{aligned}
$$

This implies

$$
\begin{aligned}
v^{\infty}(b, f) & =\inf \left\{v^{5}(b, f), v^{4}(b, f), v^{4}(b, f), v^{3}(b, f)\right\} \\
& =\inf \{0.9,0.8,0.7,0.7\}=0.7>0
\end{aligned}
$$

That is, there exist a non-zero path between $b$ and $f$. Therefore, $\mathscr{G}=(\mathscr{A}, \mathscr{B})$ in Fig. 5, is connected Pythagorean fuzzy graph. 
Theorem 2.2 The maximal product $\mathscr{G}_{1} * \mathscr{G}_{2}$ of two connected PFGs is always a connected PFG.

Proof Let $\mathscr{G}_{1}=\left(\mathscr{A}_{1}, \mathscr{B}_{1}\right)$ and $\mathscr{G}_{2}=\left(\mathscr{A}_{2}, \mathscr{B}_{2}\right)$ be two connected PFGs with underlying crisp graph $G_{1}=\left(V_{1}, E_{1}\right)$ and $G_{2}=\left(V_{2}, E_{2}\right)$, respectively. Let $V_{1}=\left\{u_{1}, u_{2}, \ldots, u_{m}\right\}$ and $V_{2}=\left\{v_{1}, v_{2}, \ldots, v_{n}\right\}$. Then $\mu_{1}^{\infty}\left(u_{i} u_{j}\right)>0, v_{1}^{\infty}\left(u_{i} u_{j}\right)$ $>0$ for all $u_{i}, u_{j} \in V_{1}$ and $\mu_{2}^{\infty}\left(v_{i} v_{j}\right)>0, v_{2}^{\infty}\left(v_{i} v_{j}\right)>0$ for all $v_{i}, v_{j} \in V_{2}$.

The maximal product of $\mathscr{G}_{1}=\left(\mathscr{A}_{1}, \mathscr{B}_{1}\right)$ and $\mathscr{G}_{2}=$ $\left(\mathscr{A}_{2}, \mathscr{B}_{2}\right)$ can be taken as $\mathscr{G}=(\mathscr{A}, \mathscr{B})$. Consider the $k$ subgraphs of $\mathscr{G}=(\mathscr{A}, \mathscr{B})$ with the vertex sets $V_{2}=$ $\left\{u_{i} v_{1}, u_{i} v_{2}, \ldots, u_{i} v_{n}\right\}$ for $i=1,2, \ldots, m$. Each of these subgraphs of $\mathscr{G}=(\mathscr{A}, \mathscr{B})$ is connected as $u_{i}^{\prime} s$ are same.

Since $\mathscr{G}_{1}=\left(\mathscr{A}_{1}, \mathscr{B}_{1}\right)$ and $\mathscr{G}_{2}=\left(\mathscr{A}_{2}, \mathscr{B}_{2}\right)$ are connected, each $u_{i}$ and $v_{i}$ are adjacent to at least one of the vertices in $V_{1}$ and $V_{2}$. Therefore, there exists at least one edge between any pair of the above $k$ subgraphs.

Thus, we have

$$
\begin{aligned}
& \mu^{\infty}\left(\left(u_{i} v_{j}\right)\left(u_{k}, v_{l}\right)\right)>0, \\
& v^{\infty}\left(\left(u_{i} v_{j}\right)\left(u_{k}, v_{l}\right)\right)>0 \quad \text { for all }\left(u_{i}, v_{j}\right)\left(u_{k}, v_{l}\right) \in E .
\end{aligned}
$$

Hence, $\mathscr{G}=(\mathscr{A}, \mathscr{B})$ is a connected PFG.

Definition 2.4 A Pythagorean fuzzy graph $\mathscr{G}=(\mathscr{A}, \mathscr{B})$ is said to be a complete Pythagorean fuzzy graph on underlying crisp graph $G=(V, E)$ if

$$
\begin{aligned}
\mu_{\mathscr{B}}(u v) & =\mu_{\mathscr{A}}(u) \wedge \mu_{\mathscr{A}}(v), \\
v_{\mathscr{B}}(u v) & =v_{\mathscr{A}}(u) \vee v_{\mathscr{A}}(v) \quad \text { for all } \mathrm{u}, \mathrm{v} \in \mathrm{V} .
\end{aligned}
$$

Example 2.3 Consider a graph $G=(V, E)$ where $V=$ $\{a, b, c, d, e\}$ and $E=\{a b, a c, a d, a e, b c, b d, b e, c d, c e$, $d e\}$. Let $\mathscr{A}$ and $\mathscr{B}$ be Pythagorean fuzzy vertex set and Pythagorean fuzzy edge set defined on $V$ and $V \times V$, respectively.

$$
\begin{aligned}
\mathscr{A}= & \left\langle\left(\frac{a}{0.25}, \frac{b}{0.8}, \frac{c}{0.65}, \frac{d}{0.4}, \frac{e}{0.9}\right),\right. \\
& \left.\times\left(\frac{a}{0.8}, \frac{b}{0.7}, \frac{c}{0.6}, \frac{d}{0.7}, \frac{e}{0.4}\right)\right\rangle \text { and } \\
\mathscr{B}= & \left\langle\left(\frac{a b}{0.4}, \frac{a c}{0.25}, \frac{a d}{0.4}, \frac{a e}{0.4}, \frac{b c}{0.4}, \frac{b d}{0.4}, \frac{b e}{0.4}, \frac{c d}{0.4}, \frac{c e}{0.4}, \frac{d e}{0.4}\right),\right. \\
& \left.\times\left(\frac{a b}{0.7}, \frac{a c}{0.8}, \frac{a d}{0.7}, \frac{a e}{0.7}, \frac{b c}{0.7}, \frac{b d}{0.4}, \frac{b e}{0.4}, \frac{c d}{0.4}, \frac{c e}{0.4}, \frac{d e}{0.4}\right)\right\rangle .
\end{aligned}
$$

By routine calculation, one can see from Fig. 6 that it is a complete Pythagorean fuzzy graph.

Remark 2.2 If two Pythagorean fuzzy graphs are complete, their maximal product may not be a complete PFG, as it can be seen in the following example. Consider two PFGs $\mathscr{G}_{1}=\left(\mathscr{A}_{1}, \mathscr{B}_{1}\right)$ and $\mathscr{G}_{2}=\left(\mathscr{A}_{2}, \mathscr{B}_{2}\right)$ on $V_{1}=\left\{u_{1}, u_{2}\right\}$ and

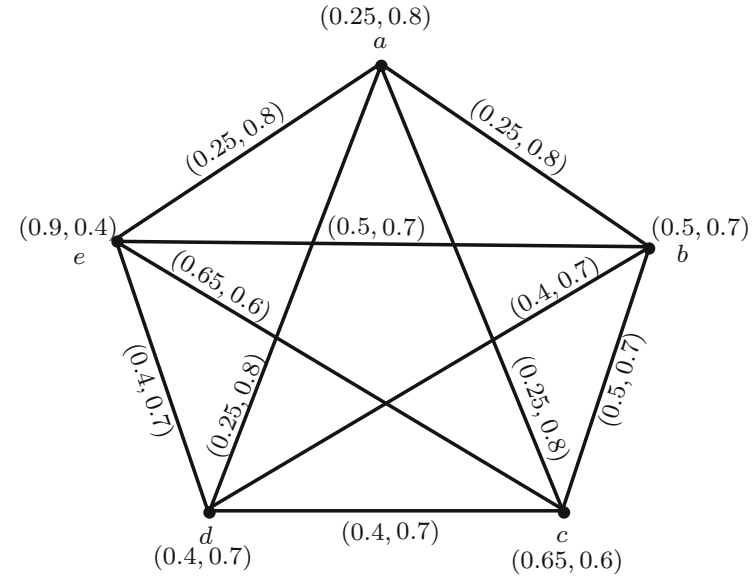

Fig. 6 Complete Pythagorean fuzzy graph

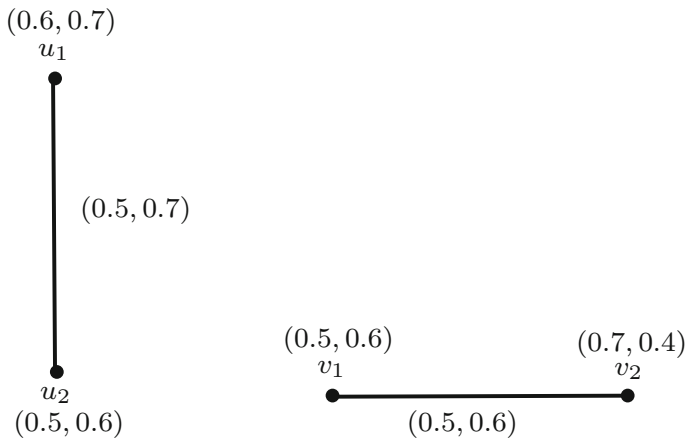

(a) $\mathscr{G}_{1}$

(b) $\mathscr{G}_{2}$

Fig. 7 Pythagorean fuzzy graphs

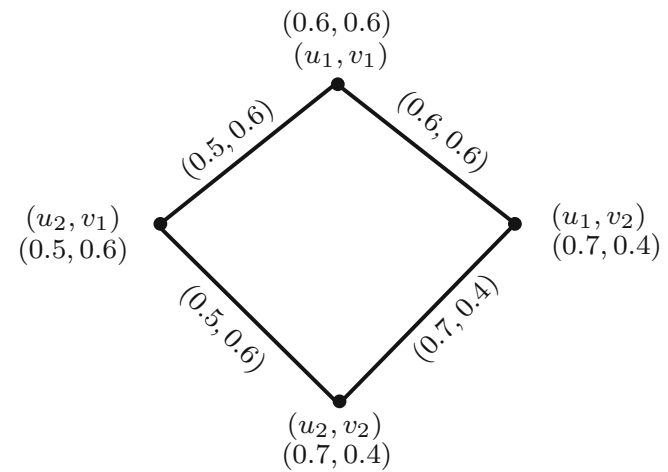

Fig. 8 Maximal product of two PFGs

$V_{2}=\left\{v_{1}, v_{2}\right\}$, respectively, as shown in Fig. 7. Their maximal product $\mathscr{G}_{1} * \mathscr{G}_{2}$ is shown in Fig. 8 .

By routine calculation, one can see from Fig. 7 that $\mathscr{G}_{1}$ and $\mathscr{G}_{2}$ are complete PFGs. While notice that $\mathscr{G}_{1} * \mathscr{G}_{2}$ is not a complete PFG, as the case $u_{1} u_{2} \in E_{1}$ and $v_{1} v_{2} \in E_{2}$ is not included in the definition of the maximal product. Further, one can notice that the maximal product of two complete PFGs is a strong PFG (Fig. 9). 


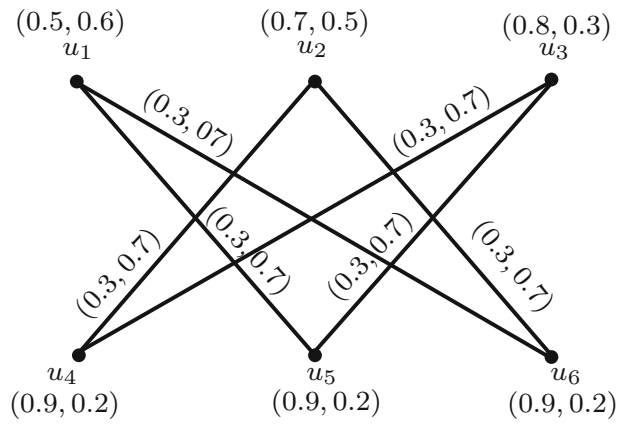

Fig. $9 \mathscr{G}=(\mathscr{A}, \mathscr{B})$

Definition 2.5 Let $\mathscr{G}=(\mathscr{A}, \mathscr{B})$ be a Pythagorean fuzzy graph on underlying crisp graph $G=(V, E)$. If

$$
\begin{aligned}
d_{\mu}(u) & =\sum_{u, v \neq u \in V} \mu_{\mathscr{B}}(u v)=k, \\
d_{\nu}(u) & =\sum_{u, v \neq u \in V} \nu_{\mathscr{B}}(u v)=l \quad \text { for all } \mathrm{u} \in \mathrm{V},
\end{aligned}
$$

then $\mathscr{G}$ is said to be regular Pythagorean fuzzy graph of degree $(k, l)$ or $(k, l)$-regular PFG.

Example 2.4 Consider a graph $G=(V, E)$ where $V=$ $\left\{u_{1}, u_{2}, u_{3}, u_{4}, u_{5}, u_{6}\right\}$ and $E=\left\{u_{1} u_{5}, u_{1} u_{6}, u_{2} u_{4}\right.$, $\left.u_{2} u_{6}, u_{3} u_{4}, u_{3} u_{5}\right\}$. Let $\mathscr{A}$ and $\mathscr{B}$ be Pythagorean fuzzy vertex set and Pythagorean fuzzy edge set defined on $V$ and $V \times V$, respectively.

$$
\begin{aligned}
\mathscr{A}= & \left\langle\left(\frac{u_{1}}{0.5}, \frac{u_{2}}{0.7}, \frac{u_{3}}{0.8}, \frac{u_{4}}{0.9}, \frac{u_{5}}{0.9}, \frac{u_{6}}{0.9}\right),\right. \\
& \left.\times\left(\frac{u_{1}}{0.6}, \frac{u_{2}}{0.5}, \frac{u_{3}}{0.3}, \frac{u_{4}}{0.2}, \frac{u_{5}}{0.2}, \frac{u_{6}}{0.2}\right)\right\rangle \text { and } \\
\mathscr{B}= & \left\langle\left(\frac{u_{1} u_{5}}{0.3}, \frac{u_{1} u_{6}}{0.3}, \frac{u_{2} u_{4}}{0.3}, \frac{u_{2} u_{6}}{0.3}, \frac{u_{3} u_{4}}{0.3}, \frac{u_{3} u_{5}}{0.3}\right),\right. \\
& \left.\times\left(\frac{u_{1} u_{5}}{0.7}, \frac{u_{1} u_{6}}{0.7}, \frac{u_{2} u_{4}}{0.7}, \frac{u_{2} u_{6}}{0.7}, \frac{u_{3} u_{4}}{0.7}, \frac{u_{3} u_{5}}{0.7}\right)\right\rangle .
\end{aligned}
$$

Since $d_{\mu}\left(u_{i}\right)=0.6$ and $d_{v}\left(u_{i}\right)=1.4$ for all $\mathrm{u}_{\mathrm{i}} \in V$ and $i=1,2, \ldots, 6, \mathscr{G}$ is a regular Pythagorean fuzzy graph of degree $(0.3,0.7)$ or $(0.3,0.7)$-regular PFG.

Remark 2.3 If $\mathscr{G}_{1}=\left(\mathscr{A}_{1}, \mathscr{B}_{1}\right)$ and $\mathscr{G}_{2}=\left(\mathscr{A}_{2}, \mathscr{B}_{2}\right)$ are two regular PFGs, then their maximal product $\mathscr{G}_{1} * \mathscr{G}_{2}$ may not be regular PFG as it can be seen in this example. Consider two PFGs $\mathscr{G}_{1}=\left(\mathscr{A}_{1}, \mathscr{B}_{1}\right)$ and $\mathscr{G}_{2}=\left(\mathscr{A}_{2}, \mathscr{B}_{2}\right)$ on $V_{1}=\{a, b\}$ and $V_{2}=\{c, d, e, f\}$, respectively, as shown in Fig. 10. Their maximal product $\mathscr{G}_{1} * \mathscr{G}_{2}$ is shown in Fig. 11 .

By routine calculation, one can see from Fig. 10 that $\mathscr{G}_{1}$ and $\mathscr{G}_{2}$ are regular PFGs. While notice that

$$
\left(d_{\mu}\right) \mathscr{G}_{1 * \mathscr{G}_{2}}(a, c)=\left\{\mu_{\mathscr{A}_{1}}(a) \vee \mu_{\mathscr{B}_{2}}(c d)\right\}
$$

$$
\begin{aligned}
& \quad+\left\{\mu_{\mathscr{A}_{1}}(a) \vee \mu_{\mathscr{B}_{2}}(c f)\right\}+\left\{\mu_{\mathscr{B}_{1}}(a b) \vee \mu_{\mathscr{A}_{2}}(c)\right\}=1.4, \\
& \left(d_{v}\right) \mathscr{G}_{1} * \mathscr{G}_{2}(a, c)=\left\{v_{\mathscr{A}_{1}}(a) \wedge v_{\mathscr{B}_{2}}(c d)\right\} \\
& \quad+\left\{v_{\mathscr{A}_{1}}(a) \wedge v_{\mathscr{B}_{2}}(c f)\right\}+\left\{v_{\mathscr{B}_{1}}(a b) \wedge v_{\mathscr{A}_{2}}(c)\right\}=1.8, \\
& \left(d_{\mu}\right) \mathscr{G}_{1} * \mathscr{G}_{2}(b, f)=\left\{\mu_{\mathscr{A}_{1}}(b) \vee \mu_{\mathscr{B}_{2}}(f c)\right\} \\
& \quad+\left\{\mu_{\mathscr{A}_{1}}(b) \vee \mu_{\mathscr{B}_{2}}(f e)\right\}+\left\{\mu_{\mathscr{B}_{1}}(a b) \vee \mu_{\mathscr{A}_{2}}(f)\right\}=1.2, \\
& \left(d_{v}\right) \mathscr{G}_{\mathscr{G}^{*} \mathscr{G}_{2}}(b, f)=\left\{v_{\mathscr{A}_{1}}(b) \wedge v_{\mathscr{B}_{2}}(f c)\right\} \\
& \quad+\left\{v_{\mathscr{A}_{1}}(b) \wedge v_{\mathscr{B}_{2}}(f e)\right\}+\left\{v_{\mathscr{B}_{1}}(a b) \wedge v_{\mathscr{A}_{2}}(f)\right\}=1.7 .
\end{aligned}
$$

That is, $(d)_{\mathscr{G}_{1} * \mathscr{G}_{2}}(a, c) \neq(d) \mathscr{G}_{1} * \mathscr{G}_{2}(b, f)$. Therefore, $\mathscr{G}_{1} * \mathscr{G}_{2}$ is not regular PFG.

Definition 2.6 Let $\mathscr{G}=(\mathscr{A}, \mathscr{B})$ be a Pythagorean fuzzy graph on underlying crisp graph $G=(V, E)$. Then, $\mathscr{G}$ is said to be a partially regular Pythagorean fuzzy graph if $G=(V, E)$ is a regular graph.

Example 2.5 Consider a Pythagorean fuzzy graph $\mathscr{G}=$ $(\mathscr{A}, \mathscr{B})$ as displayed in the Fig. 12.

Since $d_{\mu}(a)=1.65 \neq 2=d_{\mu}(a)$ and $d_{\nu}(a)=2.35 \neq$ $1.8=d_{v}(b)$. Hence, $\mathscr{G}$ is not a regular Pythagorean fuzzy graph but $G$ is a regular graph as the degree of each vertex is equal. Thus, $\mathscr{G}$ is a partially regular Pythagorean fuzzy graph.

The following theorems explain the conditions for the maximal product of two regular PFGs to be regular.

Theorem 2.3 If $\mathscr{G}_{1}=\left(\mathscr{A}_{1}, \mathscr{B}_{1}\right)$ is a partially regular $P F G$ and $\mathscr{G}_{2}=\left(\mathscr{A}_{2}, \mathscr{B}_{2}\right)$ is a PFG such that $\mu_{\mathscr{A}_{1}} \leq \mu_{\mathscr{B}_{2}}, v_{\mathscr{A}_{1}} \geq$ $v_{\mathscr{B}_{2}}$ and $\mu_{\mathscr{A}_{2}}, v_{\mathscr{A}_{2}}$ are constant functions of values $c_{1}$ and $c_{2}$, respectively, then their maximal product is regular if and only if $\mathscr{G}_{2}=\left(\mathscr{A}_{2}, \mathscr{B}_{2}\right)$ is regular $P F G$.

Proof Let $\mathscr{G}_{1}=\left(\mathscr{A}_{1}, \mathscr{B}_{1}\right)$ be a partially regular PFG such that $G_{1}=\left(V_{1}, E_{1}\right)$ is r-regular and $\mathscr{G}_{2}=\left(\mathscr{A}_{2}, \mathscr{B}_{2}\right)$ be any PFG with $\mu_{\mathscr{A}_{1}} \leq \mu_{\mathscr{B}_{2}}, v_{\mathscr{A}_{1}} \geq v_{\mathscr{B}_{2}}$ then $\mu_{\mathscr{A}_{2}} \geq \mu_{\mathscr{B}_{1}}$, $v_{\mathscr{A}_{2}} \leq v_{\mathscr{B}_{1}}$ and $\mu_{\mathscr{A}_{2}}, v_{\mathscr{A}_{2}}$ are constant functions of values $c_{1}$ and $c_{2}$, respectively.

Now assume that $\mathscr{G}_{2}=\left(\mathscr{A}_{1}, \mathscr{B}_{1}\right)$ is a $(k, l)$-regular PFG. Then, the degree of any vertex in maximal product is given by,

$$
\begin{aligned}
\left(d_{\mu}\right) \mathscr{G}_{1} * \mathscr{G}_{2}\left(u_{1}, u_{2}\right)= & \sum_{\left(u_{1}, u_{2}\right)\left(v_{1}, v_{2}\right) \in E_{1} \times E_{2}}\left(\mu_{\mathscr{B}_{1}} * \mu_{\mathscr{B}_{2}}\right)\left(\left(u_{1}, u_{2}\right)\left(v_{1}, v_{2}\right)\right) \\
= & \sum_{u_{1}=v_{1}, u_{2} v_{2} \in E_{2}} \mu_{\mathscr{A}_{1}}\left(u_{1}\right) \vee \mu_{\mathscr{B}_{2}}\left(u_{2} v_{2}\right) \\
& +\sum_{u_{2}=v_{2}, u_{1} v_{1} \in E_{1}} \mu_{\mathscr{B}_{1}}\left(u_{1} v_{1}\right) \vee \mu_{\mathscr{A}_{2}}\left(u_{2}\right) \\
= & \sum_{u_{1}=v_{1}, u_{2} v_{2} \in E_{2}} \mu_{\mathscr{B}_{2}}\left(u_{2} v_{2}\right) \\
& +\sum_{u_{2}=v_{2}, u_{1} v_{1} \in E_{1}} \mu_{\mathscr{A}_{2}}\left(u_{2}\right) \\
= & \left(d_{\mu}\right) \mathscr{G}_{2}\left(u_{2}\right)+d_{G_{1}}\left(u_{1}\right) \mu_{\mathscr{A}_{2}}\left(u_{2}\right) \\
= & r c_{1}+k
\end{aligned}
$$


Fig. 10 Pythagorean fuzzy graphs

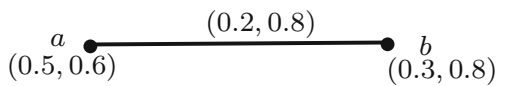

(a) $\mathscr{G}_{1}$

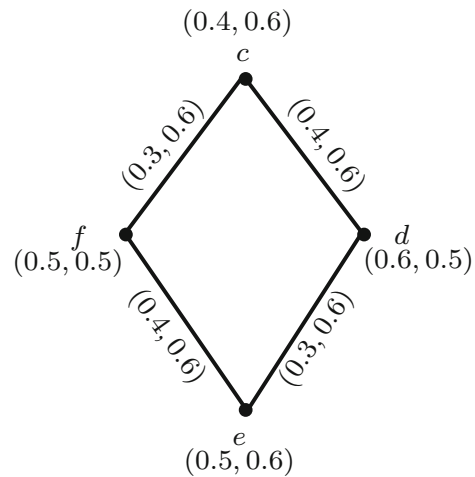

(b) $\mathscr{G}_{2}$

Fig. 11 Maximal product of two PFGs

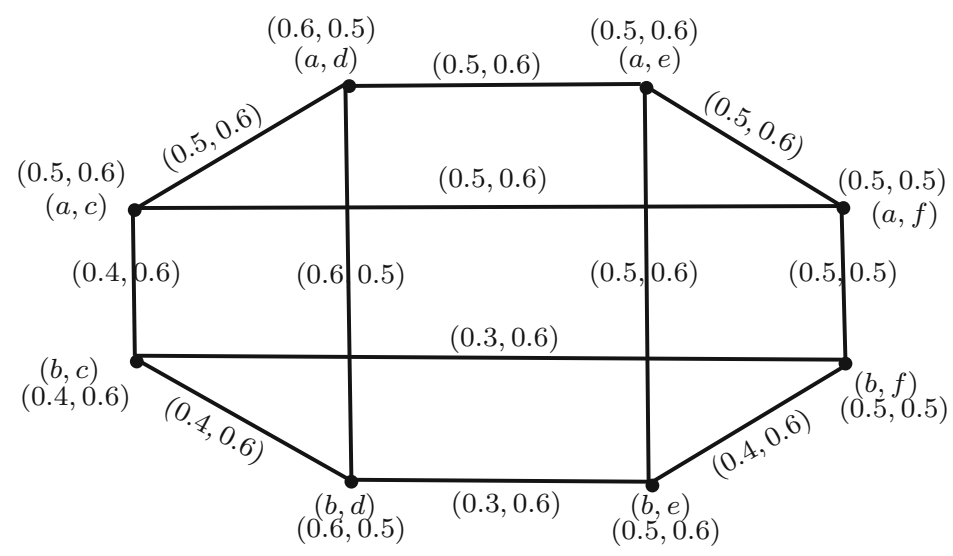

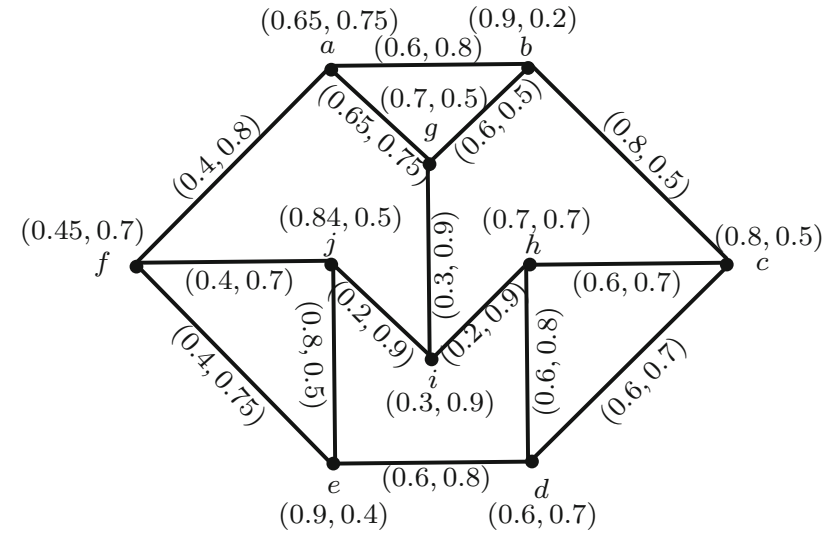

Fig. 12 Pythagorean fuzzy graph

$$
\begin{aligned}
\left(d_{v}\right) \mathscr{G}_{1} * \mathscr{G}_{2}\left(u_{1}, u_{2}\right)= & \sum_{\left(u_{1}, u_{2}\right)\left(v_{1}, v_{2}\right) \in E_{1} \times E_{2}}\left(v_{\mathscr{B}} * v_{\mathscr{B}_{2}}\right)\left(\left(u_{1}, u_{2}\right)\left(v_{1}, v_{2}\right)\right) \\
= & \sum_{u_{1}=v_{1}, u_{2} v_{2} \in E_{2}} v_{\mathscr{A}_{1}}\left(u_{1}\right) \wedge v_{\mathscr{B}_{2}}\left(u_{2} v_{2}\right) \\
& +\sum_{u_{2}=v_{2}, u_{1} v_{1} \in E_{1}} v_{\mathscr{B}_{1}}\left(u_{1} v_{1}\right) \wedge v_{\mathscr{A}_{2}}\left(u_{2}\right) \\
= & \sum_{u_{1}=v_{1}, u_{2} v_{2} \in E_{2}} v_{\mathscr{B}_{2}}\left(u_{2} v_{2}\right) \\
& +\sum_{u_{2}=v_{2}, u_{1} v_{1} \in E_{1}} v_{\mathscr{A}_{2}}\left(u_{2}\right)
\end{aligned}
$$

$$
\begin{aligned}
& =\left(d_{\mu}\right) \mathscr{G}_{2}\left(u_{2}\right)+d_{G_{1}}\left(u_{1}\right) v_{\mathscr{A}_{2}}\left(u_{2}\right) \\
& =r c_{2}+l .
\end{aligned}
$$

This is constant for all vertices in $V_{1} \times V_{2}$. Hence, $\mathscr{G}_{1} * \mathscr{G}_{2}$ is a regular PFG.

Conversely, assume that $\mathscr{G}_{1} * \mathscr{G}_{2}$ is a regular PFG. Then, for any two vertices $\left(u_{1}, v_{1}\right)$ and $\left(u_{2}, v_{2}\right)$ in $V_{1} \times V_{2}$,

$$
\begin{aligned}
& \left(d_{\mu}\right)_{\mathscr{G}_{1} * \mathscr{G}_{2}}\left(u_{1}, v_{1}\right)=\left(d_{\mu}\right) \mathscr{G}_{1} * \mathscr{G}_{2}\left(u_{2}, v_{2}\right) \\
& \left(d_{\mu}\right) \mathscr{G}_{2}\left(v_{1}\right)+d_{G_{1}}\left(u_{1}\right) \mu_{\mathscr{\mathscr { A } _ { 2 }}}\left(v_{1}\right)=\left(d_{\mu}\right) \mathscr{G}_{2}\left(v_{2}\right) \\
& +d_{G_{1}}\left(u_{2}\right) \mu_{\mathscr{A}_{2}}\left(v_{2}\right) \\
& r c_{1}+\left(d_{\mu}\right) \mathscr{G}_{2}\left(v_{1}\right)=r c_{1}+\left(d_{\mu}\right) \mathscr{G}_{2}\left(v_{2}\right) \\
& \left(d_{\mu}\right) \mathscr{G}_{2}\left(v_{1}\right)=\left(d_{\mu}\right) \mathscr{G}_{2}\left(v_{2}\right), \\
& \left(d_{v}\right) \mathscr{G}_{1} * \mathscr{G}_{2}\left(u_{1}, v_{1}\right)=\left(d_{v}\right) \mathscr{G}_{1} * \mathscr{G}_{2}\left(u_{2}, v_{2}\right) \\
& \left(d_{v}\right) \mathscr{G}_{2}\left(v_{1}\right)+d_{G_{1}}\left(u_{1}\right) v_{\mathscr{A}_{2}}\left(v_{1}\right)=\left(d_{v}\right) \mathscr{G}_{2}\left(v_{2}\right) \\
& +d_{G_{1}}\left(u_{2}\right) v_{\mathscr{A}_{2}}\left(v_{2}\right) \\
& r c_{2}+\left(d_{v}\right) \mathscr{G}_{2}\left(v_{1}\right)=r c_{2}+\left(d_{v}\right) \mathscr{G}_{2}\left(v_{2}\right) \\
& \left(d_{v}\right) \mathscr{G}_{2}\left(v_{1}\right)=\left(d_{v}\right) \mathscr{G}_{2}\left(v_{2}\right) \text {. }
\end{aligned}
$$

This is true for all vertices in $\mathscr{G}_{2}$. Hence, $\mathscr{G}_{2}$ is regular PFG.

Theorem 2.4 If $\mathscr{G}_{1}=\left(\mathscr{A}_{1}, \mathscr{B}_{1}\right)$ and $\mathscr{G}_{2}=\left(\mathscr{A}_{2}, \mathscr{B}_{2}\right)$ are two partially regular $P F G$ such that $\mu_{\mathscr{A}_{1}} \geq \mu_{\mathscr{B}_{2}}, \nu_{\mathscr{A}_{1}} \leq \nu_{\mathscr{B}_{2}}$, 
$\mu_{\mathscr{A}_{2}} \geq \mu_{\mathscr{B}_{1}}, v_{\mathscr{A}_{2}} \leq v_{\mathscr{B}_{1}}$ and $\mu_{\mathscr{A}_{2}}, v_{\mathscr{A}_{2}}$ are constant functions of values $c_{1}$ and $c_{2}$, respectively, then their maximal product is regular if and only if $\mu_{\mathscr{A}_{1}}$ and $\nu_{\mathscr{A}_{1}}$ are constant functions.

Proof Let $\mathscr{G}_{1}=\left(\mathscr{A}_{1}, \mathscr{B}_{1}\right)$ and $\mathscr{G}_{2}=\left(\mathscr{A}_{2}, \mathscr{B}_{2}\right)$ are two partially regular PFG such that $\mu_{\mathscr{A}_{1}} \geq \mu_{\mathscr{B}_{2}}, v_{\mathscr{A}_{1}} \leq v_{\mathscr{B}_{2}}$, $\mu_{\mathscr{A}_{2}} \geq \mu_{\mathscr{B}_{1}}, v_{\mathscr{A}_{2}} \leq v_{\mathscr{B}_{1}}$ and $\mu_{\mathscr{A}_{2}}, v_{\mathscr{A}_{2}}$ are constant functions of values $c_{1}$ and $c_{2}$, respectively, with $G_{i}$ is $r_{i}$-regular, $i=$ 1,2 . Now assume that $\mu_{\mathscr{A}_{1}}$ and $v_{\mathscr{A}_{1}}$ are constant functions of values $c_{3}$ and $c_{4}$, respectively. Then, the degree of any vertex in maximal product is given by,

$$
\begin{aligned}
& \left(d_{\mu}\right) \mathscr{G}_{1} * \mathscr{G}_{2}\left(u_{1}, u_{2}\right)=\sum_{\left(u_{1}, u_{2}\right)\left(v_{1}, v_{2}\right) \in E_{1} \times E_{2}}\left(\mu_{\mathscr{B}} * \mu_{\mathscr{B}_{2}}\right)\left(\left(u_{1}, u_{2}\right)\left(v_{1}, v_{2}\right)\right) \\
& =\sum_{u_{1}=v_{1}, u_{2} v_{2} \in E_{2}} \mu_{\mathscr{A}_{1}}\left(u_{1}\right) \vee \mu_{\mathscr{B}_{2}}\left(u_{2} v_{2}\right) \\
& +\sum_{u_{2}=v_{2}, u_{1} v_{1} \in E_{1}} \mu_{\mathscr{B}}\left(u_{1} v_{1}\right) \vee \mu_{\mathscr{A}_{2}}\left(u_{2}\right) \\
& =\sum_{u_{1}=v_{1}, u_{2} v_{2} \in E_{2}} \mu_{\mathscr{A}_{1}}\left(u_{1}\right) \\
& +\sum_{u_{2}=v_{2}, u_{1} v_{1} \in E_{1}} \mu_{\mathscr{A}_{2}}\left(u_{2}\right) \\
& =d_{G_{2}}\left(u_{2}\right) \mu_{\mathscr{A}_{1}}\left(u_{1}\right)+d_{G_{1}}\left(u_{1}\right) \mu_{\mathscr{A}_{2}}\left(u_{2}\right) \\
& =r_{1} c_{1}+r_{2} c_{3} \text {, } \\
& \left(d_{v}\right) \mathscr{G}_{1} * \mathscr{G}_{2}\left(u_{1}, u_{2}\right)=\sum_{\left(u_{1}, u_{2}\right)} \sum_{\left(v_{1}, v_{2}\right) \in E_{1} \times E_{2}}\left(v_{\mathscr{B}_{1}} * v_{\mathscr{B}_{2}}\right)\left(\left(u_{1}, u_{2}\right)\left(v_{1}, v_{2}\right)\right) \\
& =\sum_{u_{1}=v_{1}, u_{2} v_{2} \in E_{2}} v_{\mathscr{A}_{1}}\left(u_{1}\right) \wedge v_{\mathscr{B}}{ }_{2}\left(u_{2} v_{2}\right) \\
& +\sum_{u_{2}=v_{2}, u_{1} v_{1} \in E_{1}} v_{\mathscr{B}}\left(u_{1} v_{1}\right) \wedge v_{\mathscr{A}_{2}}\left(u_{2}\right) \\
& =\sum_{u_{1}=v_{1}, u_{2} v_{2} \in E_{2}} v_{\mathscr{A}_{1}}\left(u_{1}\right) \\
& +\sum_{u_{2}=v_{2}, u_{1} v_{1} \in E_{1}} v_{\mathscr{A}_{2}}\left(u_{2}\right) \\
& =d_{G_{2}}\left(u_{2}\right) v_{\mathscr{A}_{1}}\left(u_{1}\right)+d_{G_{1}}\left(u_{1}\right) v_{\mathscr{A}_{2}}\left(u_{2}\right) \\
& =r_{1} c_{2}+r_{2} c_{4} \text {. }
\end{aligned}
$$

This is constant for all vertices in $V_{1} \times V_{2}$. Hence, $\mathscr{G}_{1} * \mathscr{G}_{2}$ is a regular PFG.

Conversely, assume that $\mathscr{G}_{1} * \mathscr{G}_{2}$ is a regular PFG. Then, for any two vertices $\left(u_{1}, v_{1}\right)$ and $\left(u_{2}, v_{2}\right)$ in $V_{1} \times V_{2}$,

$$
\begin{aligned}
\left(d_{\mu}\right) \mathscr{G}_{1} * \mathscr{G}_{2}\left(u_{1}, v_{1}\right)= & \left(d_{\mu}\right) \mathscr{G}_{1} * \mathscr{G}_{2}\left(u_{2}, v_{2}\right) \\
d_{G_{1}}\left(u_{1}\right) \mu_{\mathscr{A}_{2}}\left(v_{1}\right)+d_{G_{2}}\left(v_{1}\right) \mu_{\mathscr{A}_{1}}\left(u_{1}\right)= & d_{G_{1}}\left(u_{2}\right) \mu_{\mathscr{A}_{2}}\left(v_{2}\right) \\
& +d_{G_{2}}\left(v_{2}\right) \mu_{\mathscr{A}_{1}}\left(u_{2}\right) \\
r c_{1}+r_{2} \mu_{\mathscr{A}_{1}}\left(u_{1}\right)= & r c_{1}+r_{2} \mu_{\mathscr{A}_{1}}\left(u_{2}\right) \\
\mu_{\mathscr{A}_{1}}\left(u_{1}\right)= & \mu_{\mathscr{A}_{1}}\left(u_{2}\right), \\
\left(d_{v}\right) \mathscr{G}_{1} * \mathscr{G}_{2}\left(u_{1}, v_{1}\right)= & \left(d_{v}\right) \mathscr{G}_{1} * \mathscr{G}_{2}\left(u_{2}, v_{2}\right) \\
d_{G_{1}}\left(u_{1}\right) v_{\mathscr{A}_{2}}\left(v_{1}\right)+d_{G_{2}}\left(v_{1}\right) v_{\mathscr{A}_{1}}\left(u_{1}\right)= & d_{G_{1}}\left(u_{2}\right) v_{\mathscr{A}_{2}}\left(v_{2}\right) \\
& +d_{G_{2}}\left(v_{2}\right) v_{\mathscr{A}_{1}}\left(u_{2}\right) \\
r c_{2}+r_{2} v_{\mathscr{A}_{1}}\left(u_{1}\right)= & r c_{2}+r_{2} v_{\mathscr{A}_{1}}\left(u_{2}\right) \\
v_{\mathscr{A}_{1}}\left(u_{1}\right)= & v_{\mathscr{A}_{1}}\left(u_{2}\right) .
\end{aligned}
$$

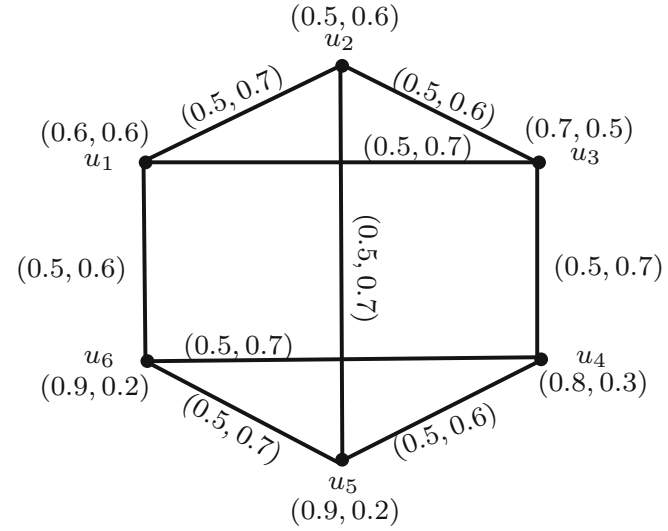

Fig. 13 Pythagorean fuzzy graph

This is true for all vertices in $\mathscr{G}_{1}$. Hence, $\mu_{\mathscr{A}_{1}}$ and $v_{\mathscr{A}_{1}}$ are constant functions.

Definition 2.7 Let $\mathscr{G}=(\mathscr{A}, \mathscr{B})$ be a Pythagorean fuzzy graph on underlying crisp graph $G=(V, E)$. Then $\mathscr{G}$ is said to be a full regular Pythagorean fuzzy graph if $\mathscr{G}$ is both regular and partially regular graph.

Example 2.6 Consider a Pythagorean fuzzy graph $\mathscr{G}=$ $(\mathscr{A}, \mathscr{B})$ as displayed in the Fig. 13.

Since $d_{\mu}\left(u_{i}\right)=1.5$ and $d_{v}\left(u_{i}\right)=2$ for all $u_{i} \in V$, where $i=1, \ldots, 6$. Hence, $\mathscr{G}$ is a regular Pythagorean fuzzy graph of degree $(1.5,2)$. Also, $G$ is a regular graph as the degree of each vertex is equal. Thus, $\mathscr{G}$ is a full regular Pythagorean fuzzy graph.

Remark 2.4 If two Pythagorean fuzzy graphs are full regular, their maximal product may not be full regular PFG as it can be seen in this example. Consider two PFGs $\mathscr{G}_{1}=\left(\mathscr{A}_{1}, \mathscr{B}_{1}\right)$ and $\mathscr{G}_{2}=\left(\mathscr{A}_{2}, \mathscr{B}_{2}\right)$ on $V_{1}=\{a, b\}$ and $V_{2}=\{c, d, e\}$, respectively, as shown in Fig. 14. Their maximal product $\mathscr{G}_{1} * \mathscr{G}_{2}$ is shown in Fig. 15.

By routine calculation, one can see that $\mathscr{G}_{1}$ and $\mathscr{G}_{2}$ are full regular PFGs. But $(d)_{\mathscr{G}_{1} * \mathscr{G}_{2}}(a, d) \neq(d) \mathscr{G}_{1} * \mathscr{G}_{2}(b, d)$. Hence, $\mathscr{G}_{1} * \mathscr{G}_{2}$ is not full regular PFG.

Remark 2.5 The maximal product of two regular PFGs on complete graphs is partially regular. Consider two PFGs $\mathscr{G}_{1}=$ $\left(\mathscr{A}_{1}, \mathscr{B}_{1}\right)$ and $\mathscr{G}_{2}=\left(\mathscr{A}_{2}, \mathscr{B}_{2}\right)$ on $V_{1}=\left\{u_{1}, v_{1}\right\}$ and $V_{2}=$ $\left\{u_{2}, v_{2}\right\}$, respectively, as shown in Fig. 16. Their maximal product $\mathscr{G}_{1} * \mathscr{G}_{2}$ is shown in Fig. 17.

By routine calculation, one can see that $\mathscr{G}_{1}$ and $\mathscr{G}_{2}$ are regular PFGs. But $(d)_{\mathscr{G}_{1} * \mathscr{G}_{2}}\left(u_{1}, u_{2}\right) \neq(d)_{\mathscr{G}_{1} * \mathscr{G}_{2}}\left(v_{1}, v_{2}\right)$. Hence, $\mathscr{G}_{1} * \mathscr{G}_{2}$ is a partially regular PFG as crisp graph is regular. 
Fig. 14 Pythagorean fuzzy graphs

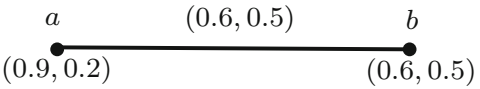

(a) $\mathscr{G}_{1}$

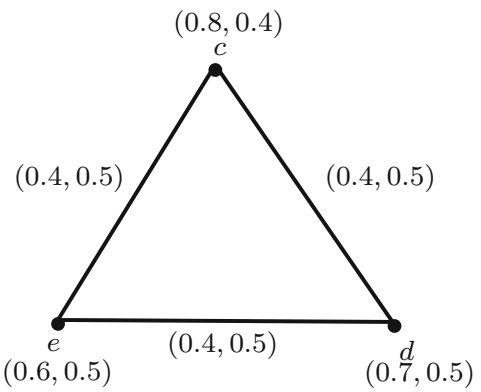

(b) $\mathscr{G}_{2}$
Fig. 15 Maximal product of two PFGs
Fig. 16 Pythagorean fuzzy graph

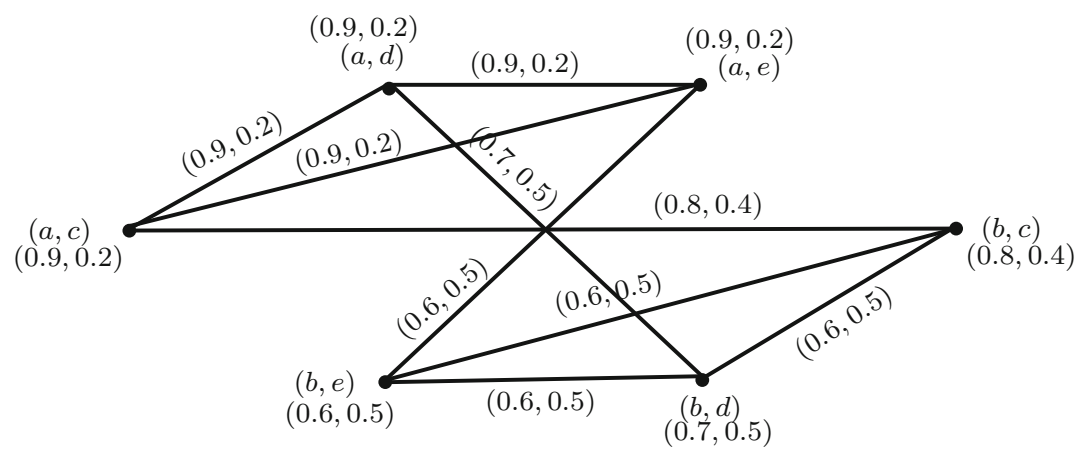

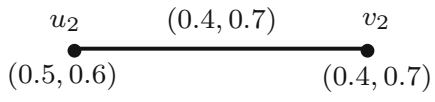

(b) $\mathscr{G}_{2}$

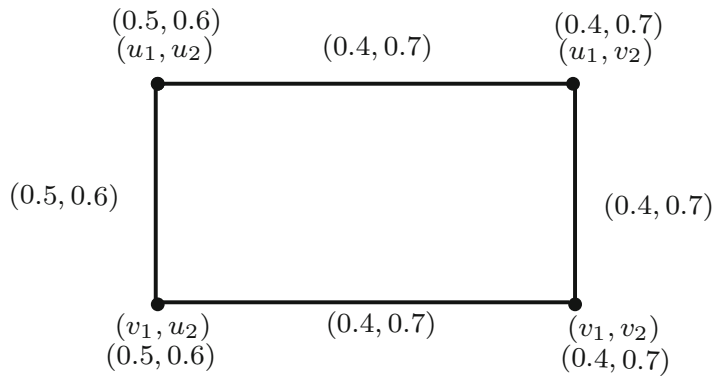

Fig. 17 Maximal product of two PFGs

\section{Regular residue product in Pythagorean fuzzy graphs}

Definition 3.1 Let $\mathscr{G}_{1}=\left(\mathscr{A}_{1}, \mathscr{B}_{1}\right)$ and $\mathscr{G}_{2}=\left(\mathscr{A}_{2}, \mathscr{B}_{2}\right)$ be two PFGs of the graphs $G_{1}=\left(V_{1}, E_{1}\right)$ and $G_{2}=\left(V_{2}, E_{2}\right)$, respectively. The residue product of $\mathscr{G}_{1}$ and $\mathscr{G}_{2}$ is denoted by $\mathscr{G}_{1} \cdot \mathscr{G}_{2}=\left(\mathscr{A}_{1} \cdot \mathscr{A}_{2}, \mathscr{B}_{1} \cdot \mathscr{B}_{2}\right)$ and defined as:
(i) $\left\{\begin{array}{l}\left(\mu_{\mathscr{A}_{1}} \cdot \mu_{\mathscr{A}_{2}}\right)\left(u_{1}, u_{2}\right)=\mu_{\mathscr{A}_{1}}\left(u_{1}\right) \vee \mu_{\mathscr{A}_{2}}\left(u_{2}\right) \\ \left(v_{\mathscr{A}_{1}} \cdot v_{\mathscr{L}_{2}}\right)\left(u_{1}, u_{2}\right)=v_{\mathscr{A}_{1}}\left(u_{1}\right) \wedge v_{\mathscr{A}_{2}}\left(u_{2}\right) \\ \quad \text { for all }\left(u_{1}, u_{2}\right) \in V_{1} \times V_{2},\end{array}\right.$
(ii) $\left\{\begin{array}{l}\left(\mu_{\mathscr{B}_{1}} \cdot \mu_{\mathscr{B}_{2}}\right)\left(u_{1}, u_{2}\right)\left(v_{1}, v_{2}\right)=\mu_{\mathscr{B}_{1}}\left(u_{1} v_{1}\right) \\ \left(v_{\mathscr{B}_{1}} \cdot v_{\mathscr{B}_{2}}\right)\left(u_{1}, u_{2}\right)\left(v_{1}, v_{2}\right)=v_{\mathscr{B}_{1}}\left(u_{1} v_{1}\right) \\ \quad \text { for all } u_{1} v_{1} \in E_{1}, u_{2} \neq v_{2} .\end{array}\right.$

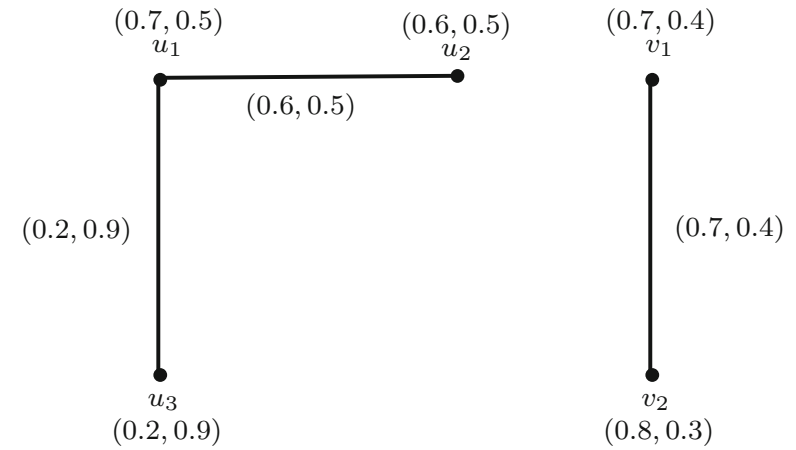

(a) $\mathscr{G}_{1}$

Fig. 18 Pythagorean fuzzy graphs

Remark 3.1 In general, the residue product of two strong PFGs may not be strong PFG as it is explained in this example. Consider two Pythagorean fuzzy graphs $\mathscr{G}_{1}=\left(\mathscr{A}_{1}, \mathscr{B}_{1}\right)$ and $\mathscr{G}_{2}=\left(\mathscr{A}_{2}, \mathscr{B}_{2}\right)$ as shown in Fig. 18.

Here $\mu_{\mathscr{B}_{1}}\left(u_{1} u_{2}\right)=\mu_{\mathscr{A}_{1}}\left(u_{1}\right) \wedge \mu_{\mathscr{A}_{1}}\left(u_{2}\right), v_{\mathscr{B}_{1}}\left(u_{1} u_{2}\right)=$ $v_{\mathscr{A}_{1}}\left(u_{1}\right) \vee v_{\mathscr{A}_{1}}\left(u_{2}\right)$ for all $u_{1} u_{2} \in E_{1}$ and $\mu_{\mathscr{B}_{2}}\left(v_{1} v_{2}\right)=$ $\mu_{\mathscr{A}_{2}}\left(v_{1}\right) \wedge \mu_{\mathscr{A}_{2}}\left(v_{2}\right), v_{\mathscr{B}_{2}}\left(v_{1} v_{2}\right)=v_{\mathscr{A}_{2}}\left(v_{1}\right) \vee v_{\mathscr{A}_{2}}\left(v_{2}\right)$ for all $v_{1} v_{2} \in E_{2}$. Hence, $\mathscr{G}_{1}$ and $\mathscr{G}_{2}$ are strong PFGs.

$\operatorname{But}\left(\mu_{\mathscr{B}_{1}} \cdot \mu_{\mathscr{B}_{2}}\right)\left(\left(u_{1}, v_{2}\right)\left(u_{2}, v_{1}\right)\right)=0.6 \neq \mu_{\mathscr{A}_{1}}\left(u_{1}, v_{2}\right) \wedge$ $\mu_{\mathscr{A}_{2}}\left(u_{2}, v_{1}\right)=0.7$ and $\left(v_{\mathscr{B}_{1}} \cdot v_{\mathscr{B}_{2}}\right)\left(\left(u_{1}, v_{2}\right)\left(u_{2}, v_{1}\right)\right)$ 
$=0.5 \neq v_{\mathscr{A}_{1}}\left(u_{1}, v_{2}\right) \wedge v_{\mathscr{A}_{2}}\left(u_{2}, v_{1}\right)=0.4$. Thus, their residue product $\mathscr{G}_{1} \cdot \mathscr{G}_{2}$ is not a strong PFG.

The following theorem gives the condition under which the residue product is strong.

Theorem 3.1 The residue product of a strong $P F G \mathscr{G}_{1}=$ $\left(\mathscr{A}_{1}, \mathscr{B}_{1}\right)$ with any PFG $\mathscr{G}_{2}=\left(\mathscr{A}_{2}, \mathscr{B}_{2}\right)$ is a strong PFG if $\mu_{\mathscr{A}_{1}} \geq \mu_{\mathscr{A}_{2}}, v_{\mathscr{A}_{1}} \leq v_{\mathscr{A}_{2}}$.

Proof Let $\mathscr{G}_{1}=\left(\mathscr{A}_{1}, \mathscr{B}_{1}\right)$ be a strong PFG and $\mathscr{G}_{2}=\left(\mathscr{A}_{2}\right.$, $\left.\mathscr{B}_{2}\right)$ be any PFG with $\mu_{\mathscr{A}_{1}} \geq \mu_{\mathscr{A}_{2}}, v_{\mathscr{A}_{1}} \leq v_{\mathscr{A}_{2}}$. Then

$\mu_{\mathscr{B}_{1}}\left(u_{1} u_{2}\right)=\mu_{\mathscr{A}_{1}}\left(u_{1}\right) \wedge \mu_{\mathscr{A}_{1}}\left(u_{2}\right)$,

$\nu_{\mathscr{B}_{1}}\left(u_{1} u_{2}\right)=\mu_{\mathscr{A}_{1}}\left(u_{1}\right) \vee \mu_{\mathscr{A}_{1}}\left(u_{2}\right)$ for all $u_{1} u_{2} \in E_{1}$.

By definition of residue product, we have

If $u_{1} u_{2} \in E_{1}$ and $\mathrm{v}_{1} \neq \mathrm{v}_{2}$, then

$$
\begin{aligned}
\left(\mu \mathscr{B}_{1}\right. & \left.\cdot \mu_{\mathscr{B}_{2}}\right)\left(\left(u_{1}, v_{1}\right)\left(u_{2}, v_{2}\right)\right) \\
& =\mu_{\mathscr{B}_{1}}\left(u_{1} u_{2}\right) \\
& =\mu_{\mathscr{A}_{1}}\left(u_{1}\right) \wedge \mu_{\mathscr{A}_{1}}\left(u_{2}\right) \\
& =\left\{\mu_{\mathscr{A}_{1}}\left(u_{1}\right) \vee \mu_{\mathscr{A}_{2}}\left(v_{1}\right)\right\} \wedge\left\{\mu_{\mathscr{A}_{1}}\left(u_{2}\right) \vee \mu_{\mathscr{A}_{2}}\left(v_{2}\right)\right\} \\
& =\left(\mu_{\mathscr{A}_{1}} \cdot \mu_{\mathscr{A}_{2}}\right)\left(u_{1}, v_{1}\right) \wedge\left(\mu_{\mathscr{A}_{1}} \cdot \mu_{\mathscr{A}_{2}}\right)\left(u_{2}, v_{2}\right), \\
\left(v_{\mathscr{B}_{1}}\right. & \left.\cdot v_{\mathscr{B}_{2}}\right)\left(\left(u_{1}, v_{1}\right)\left(u_{2}, v_{2}\right)\right) \\
& =v_{\mathscr{B}_{1}}\left(u_{1} u_{2}\right) \\
& =v_{\mathscr{A}_{1}}\left(u_{1}\right) \vee v_{\mathscr{A}_{1}}\left(u_{2}\right) \\
& =\left\{v_{\mathscr{A}_{1}}\left(u_{1}\right) \wedge v_{\mathscr{A}_{2}}\left(v_{1}\right)\right\} \vee\left\{v_{\mathscr{A}_{1}}\left(u_{2}\right) \wedge v_{\mathscr{A}_{2}}\left(v_{2}\right)\right\} \\
& =\left(v_{\mathscr{A}_{1}} \cdot v_{\mathscr{A}_{2}}\right)\left(u_{1}, v_{1}\right) \vee\left(v_{\mathscr{A}_{1}} \cdot v_{\mathscr{A}_{2}}\right)\left(u_{2}, v_{2}\right) .
\end{aligned}
$$

Hence, $\mathscr{G}_{1} \cdot \mathscr{G}_{2}$ is a strong PFG.

Theorem 3.2 The residue product $\mathscr{G}_{1} * \mathscr{G}_{2}$ of two connected PFGs is always a connected PFG.

Proof Let $\mathscr{G}_{1}=\left(\mathscr{A}_{1}, \mathscr{B}_{1}\right)$ and $\mathscr{G}_{2}=\left(\mathscr{A}_{2}, \mathscr{B}_{2}\right)$ be two connected PFGs with underlying crisp graph $G_{1}=\left(V_{1}, E_{1}\right)$ and $G_{2}=\left(V_{2}, E_{2}\right)$, respectively. Let $V_{1}=\left\{u_{1}, u_{2}, \ldots, u_{m}\right\}$ and $V_{2}=\left\{v_{1}, v_{2}, \ldots, v_{n}\right\}$. Then $\mu_{1}^{\infty}\left(u_{i} u_{j}\right)>0$, $v_{1}^{\infty}\left(u_{i} u_{j}\right)>0$ for all $u_{i}, u_{j} \in V_{1}$ and $\mu_{2}^{\infty}\left(v_{i} v_{j}\right)>0$, $v_{2}^{\infty}\left(v_{i} v_{j}\right)>0$ for all $v_{i}, v_{j} \in V_{2}$.

The residue product of $\mathscr{G}_{1}=\left(\mathscr{A}_{1}, \mathscr{B}_{1}\right)$ and $\mathscr{G}_{2}=$ $\left(\mathscr{A}_{2}, \mathscr{B}_{2}\right)$ can be taken as $\mathscr{G}=(\mathscr{A}, \mathscr{B})$. Consider the $k$ subgraphs of $\mathscr{G}=(\mathscr{A}, \mathscr{B})$ with the vertex sets $V_{2}=$ $\left\{u_{i} v_{1}, u_{i} v_{2}, \ldots, u_{i} v_{n}\right\}$ for $i=1,2, \ldots, m$. Each of these subgraphs of $\mathscr{G}=(\mathscr{A}, \mathscr{B})$ is connected as $u_{i}^{\prime} s$ are same.

Since $\mathscr{G}_{1}=\left(\mathscr{A}_{1}, \mathscr{B}_{1}\right)$ and $\mathscr{G}_{2}=\left(\mathscr{A}_{2}, \mathscr{B}_{2}\right)$ are connected, each $u_{i}$ and $v_{i}$ are adjacent to at least one of the vertices in $V_{1}$ and $V_{2}$. Therefore, there exists at least one edge between any pair of the above $k$ subgraphs.

Thus, we have

$\mu^{\infty}\left(\left(u_{i} v_{j}\right)\left(u_{k}, v_{l}\right)\right)>0$

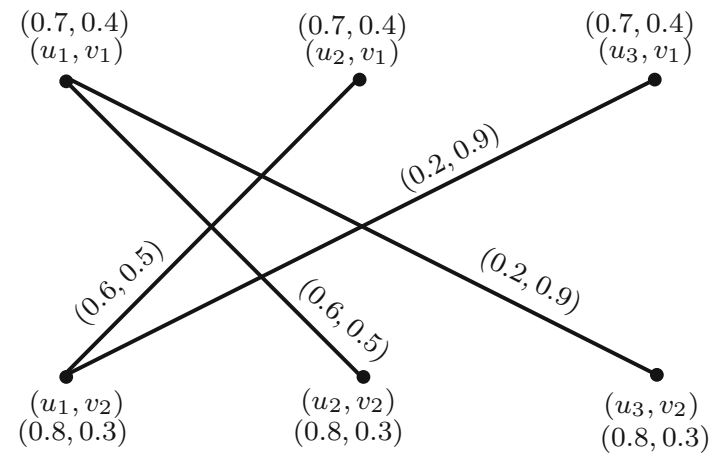

Fig. 19 Residue product of two PFGs

$v^{\infty}\left(\left(u_{i} v_{j}\right)\left(u_{k}, v_{l}\right)\right)>0$ for all $\left(u_{i}, v_{j}\right)\left(u_{k}, v_{l}\right) \in E$.

Hence, $\mathscr{G}=(\mathscr{A}, \mathscr{B})$ is a connected PFG (Fig. 19).

Remark 3.2 The residue product of two complete PFGs is not a complete PFG. We explain with an example. Consider two PFGs $\mathscr{G}_{1}=\left(\mathscr{A}_{1}, \mathscr{B}_{1}\right)$ and $\mathscr{G}_{2}=\left(\mathscr{A}_{2}, \mathscr{B}_{2}\right)$ on $V_{1}=\{a, b, c\}$ and $V_{2}=\{d, e\}$, respectively, as shown in Fig. 20. Their residue product $\mathscr{G}_{1} \cdot \mathscr{G}_{2}$ is shown in Fig. 21.

By routine calculation, one can see from Fig. 20 that $\mathscr{G}_{1}$ and $\mathscr{G}_{2}$ are complete PFGs. While notice that $\mathscr{G}_{1} \cdot \mathscr{G}_{2}$ is not a complete PFG as the only case $u_{1} u_{2} \in E_{1}$, is included in the definition of the residue product.

Remark 3.3 If $\mathscr{G}_{1}=\left(\mathscr{A}_{1}, \mathscr{B}_{1}\right)$ and $\mathscr{G}_{2}=\left(\mathscr{A}_{2}, \mathscr{B}_{2}\right)$ are two regular PFGs, then their residue product $\mathscr{G}_{1} \cdot \mathscr{G}_{2}$ may not to be regular PFG as it is explained in this example. Consider two PFGs $\mathscr{G}_{1}=\left(\mathscr{A}_{1}, \mathscr{B}_{1}\right)$ and $\mathscr{G}_{2}=\left(\mathscr{A}_{2}, \mathscr{B}_{2}\right)$ on $V_{1}=\{a, b\}$ and $V_{2}=\{c, d\}$, respectively, as shown in Fig. 22. Their residue product $\mathscr{G}_{1} \cdot \mathscr{G}_{2}$ is shown in Fig. 23.

By routine calculation, one can see from Fig. 22 that $\mathscr{G}_{1}$ and $\mathscr{G}_{2}$ are regular PFGs. While notice that $(d) \mathscr{G}_{1} \cdot \mathscr{G}_{2}(a, d) \neq$ $(d) \mathscr{G}_{1} * \mathscr{G}_{2}(a, c)$. Therefore, $\mathscr{G}_{1} \cdot \mathscr{G}_{2}$ is not regular PFG.

The following theorems explain the conditions for the residue product of two regular PFGs to be regular.

Theorem 3.3 The residue product $\mathscr{G}_{1} \cdot \mathscr{G}_{1}$ of any $P F G \mathscr{G}_{1}=$ $\left(\mathscr{A}_{1}, \mathscr{B}_{1}\right)$ with a $P F G \mathscr{G}_{2}=\left(\mathscr{A}_{2}, \mathscr{B}_{2}\right)$ such that $\left|V_{2}\right|=1$, is always a PFG with no edge.

Proof The proof follows from the Definition 3.1.

Theorem 3.4 If $\mathscr{G}_{1}=\left(\mathscr{A}_{1}, \mathscr{B}_{1}\right)$ and $\mathscr{G}_{2}=\left(\mathscr{A}_{2}, \mathscr{B}_{2}\right)$ are two PFGs such that $\left|V_{2}\right|>1$, then their residue product is regular if and only if $\mathscr{G}_{1}$ is regular.

Proof Let $\mathscr{G}_{1}=\left(\mathscr{A}_{1}, \mathscr{B}_{1}\right)$ be a $(k, l)$-regular PFG and $\mathscr{G}_{2}=$ $\left(\mathscr{A}_{2}, \mathscr{B}_{2}\right)$ be any PFG with $\left|V_{2}\right|>1$.

If $\left|V_{2}\right|>1$, then the degree of any vertex in Residue product is given by, 
Fig. 20 Pythagorean fuzzy graphs

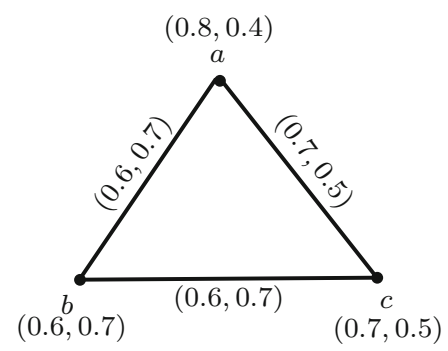

(a) $\mathscr{G}_{1}$

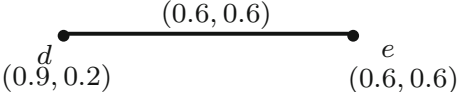

(b) $\mathscr{G}_{2}$

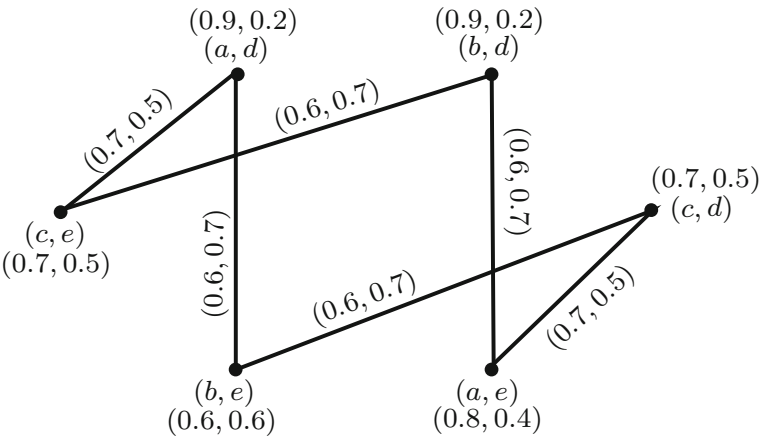

Fig. 21 Residue product of two PFGs

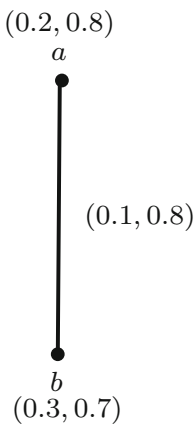

(a) $\mathscr{G}_{1}$
$(0.4,0.8)$

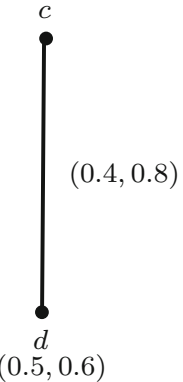

(b) $\mathscr{G}_{2}$
Fig. 22 Pythagorean fuzzy graphs

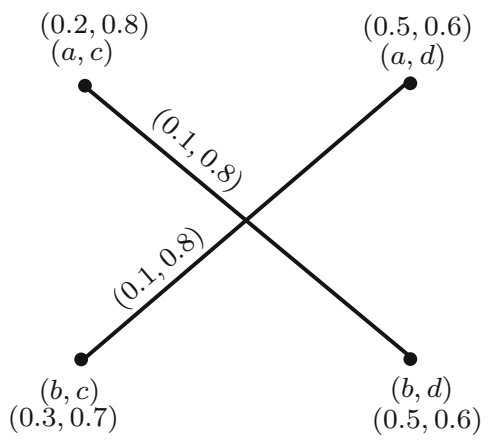

Fig. 23 Residue product of two PFGs

$$
\begin{aligned}
\left(d_{\mu}\right) \mathscr{G}_{1} \cdot \mathscr{G}_{2}\left(u_{1}, u_{2}\right) & =\sum_{u_{1} v_{1} \in E_{1}, u_{2} \neq y_{2}} \mu_{\mathscr{B}_{1}}\left(u_{1} v_{1}\right) \\
& =\left(d_{\mu}\right) \mathscr{G}_{1}\left(u_{1}\right)=k, \\
\left(d_{v}\right) \mathscr{G}_{1} \cdot \mathscr{G}_{2}\left(u_{1}, u_{2}\right) & =\sum_{u_{1} v_{1} \in E_{1}, u_{2} \neq y_{2}} v_{\mathscr{B}_{1}}\left(u_{1} v_{1}\right) \\
& =\left(d_{v}\right) \mathscr{G}_{1}\left(u_{1}\right)=l .
\end{aligned}
$$

This is a constant for all vertices in $V_{1} \times V_{2}$. Hence, $\mathscr{G}_{1} \cdot \mathscr{G}_{2}$ is a regular PFG.

Conversely, assume that $\mathscr{G}_{1} \cdot \mathscr{G}_{2}$ is a regular PFG. Then, for any two vertices $\left(u_{1}, v_{1}\right)$ and $\left(u_{2}, v_{2}\right)$ in $V_{1} \times V_{2}$,

$\left(d_{\mu}\right) \mathscr{G}_{1} \cdot \mathscr{G}_{2}\left(u_{1}, v_{1}\right)=\left(d_{\mu}\right) \mathscr{G}_{1} \cdot \mathscr{G}_{2}\left(u_{2}, v_{2}\right)$

$\left(d_{\mu}\right) \mathscr{G}_{1}\left(u_{1}\right)=\left(d_{\mu}\right) \mathscr{G}_{1}\left(u_{2}\right)$,

$\left(d_{v}\right) \mathscr{G}_{1} \cdot \mathscr{G}_{2}\left(u_{1}, v_{1}\right)=\left(d_{v}\right) \mathscr{G}_{1} \cdot \mathscr{G}_{2}\left(u_{2}, v_{2}\right)$

$\left(d_{v}\right) \mathscr{G}_{1}\left(u_{1}\right)=\left(d_{v}\right) \mathscr{G}_{1}\left(u_{2}\right)$.

This is true for all vertices in $V_{1}$. Hence, $\mathscr{G}_{1}$ is a regular PFG.

Example 3.1 Consider two PFGs $\mathscr{G}_{1}=\left(\mathscr{A}_{1}, \mathscr{B}_{1}\right)$ and $\mathscr{G}_{2}=$ $\left(\mathscr{A}_{2}, \mathscr{B}_{2}\right)$ on $\mathrm{V}_{1}=\left\{\mathrm{u}_{1}, \mathrm{u}_{2}\right\}$ and $\mathrm{V}_{2}=\left\{\mathrm{v}_{1}, \mathrm{v}_{2}, \mathrm{v}_{3}\right\}$, respectively, as shown in Fig. 24. Their residue product $\mathscr{G}_{1} \cdot \mathscr{G}_{2}$ is shown in Fig. 25.

Since $(d) \mathscr{G}_{1}\left(u_{i}\right)=(0.3,0.7)$ for $\mathrm{i}=1,2$ and $(d) \mathscr{G}_{2}\left(v_{1}\right) \neq$ $(d)_{\mathscr{G}_{2}}\left(v_{2}\right)$, therefore $\mathscr{G}_{1}=\left(\mathscr{A}_{1}, \mathscr{B}_{1}\right)$ is a regular PFG and $\mathscr{G}_{2}=\left(\mathscr{A}_{2}, \mathscr{B}_{2}\right)$ is not regular PFG with $\left|V_{2}\right|>1$. Now, (d) $\mathscr{G}_{1} \cdot \mathscr{G}_{2}\left(u_{i}, v_{j}\right)=(0.6,1.4)$ for $i=1,2$ and $j=1,2,3$. Thus, the residue product $\mathscr{G}_{1} \cdot \mathscr{G}_{2}$ is a regular PFG.

Theorem 3.5 If $\mathscr{G}_{1}=\left(\mathscr{A}_{1}, \mathscr{B}_{1}\right)$ and $\mathscr{G}_{2}=\left(\mathscr{A}_{2}, \mathscr{B}_{2}\right)$ are two PFGs such that $\mu_{\mathscr{A}_{1}} \geq \mu_{\mathscr{A}_{2}}, v_{\mathscr{A}_{1}} \leq v_{\mathscr{A}_{2}}$, then the total degree of any vertex in the residue product $\mathscr{G}_{1} \cdot \mathscr{G}_{2}$ is given as,

$$
\begin{aligned}
& \left(t d_{\mu}\right) \mathscr{G}_{1} \cdot \mathscr{G}_{2}\left(u_{i}, v_{j}\right)= \begin{cases}\left(t d_{\mu}\right) \mathscr{G}_{1}\left(u_{i}\right) & \text { if }\left|V_{1}\right|>1, \\
\mu_{\mathscr{A}_{1}}\left(u_{i}\right) & \text { if }\left|V_{1}\right|=1,\end{cases} \\
& \left(t d_{v}\right) \mathscr{G}_{1} \mathscr{G}_{2}\left(u_{i}, v_{j}\right)= \begin{cases}\left(t d_{v}\right) \mathscr{G}_{1}\left(u_{i}\right) & \text { if }\left|V_{1}\right|>1, \\
v_{\mathscr{A}_{1}}\left(u_{i}\right) & \text { if }\left|V_{1}\right|=1 .\end{cases}
\end{aligned}
$$


Fig. 24 Pythagorean fuzzy graphs
$(0.5,0.6)$

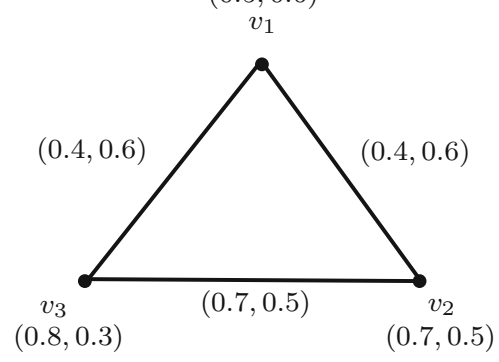

(b) $\mathscr{G}_{2}$
$(0.7,0.5)$

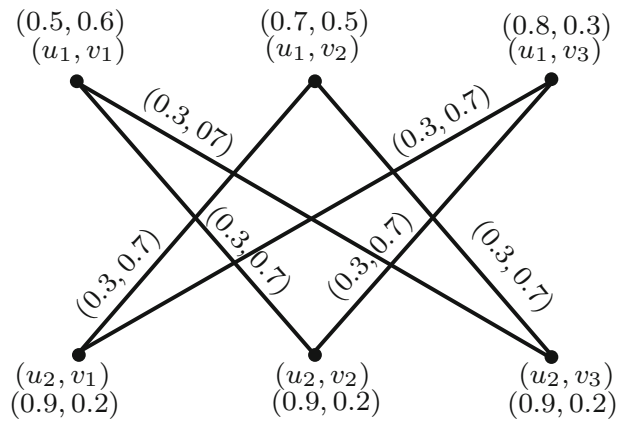

Fig. 25 Residue product of two PFGs

Proof If $\mu_{\mathscr{A}_{1}} \geq \mu_{\mathscr{A}_{2}}, v_{\mathscr{A}_{1}} \leq v_{\mathscr{A}_{2}}$ and $\left|V_{2}\right|>1$, then

$$
\begin{aligned}
& \left(t d_{\mu}\right) \mathscr{G}_{1} \cdot \mathscr{G}_{2}\left(u_{i}, v_{j}\right) \\
& =\sum_{\left(u_{i}, v_{j}\right)\left(u_{k}, v_{l}\right) \in E_{1} \times E_{2}}\left(\mu_{\mathscr{B}_{1}} \cdot \mu_{\mathscr{B}_{2}}\right)\left(\left(u_{i}, v_{j}\right)\left(u_{k}, v_{l}\right)\right) \\
& +\left(\mu_{\mathscr{A}_{1}} \cdot \mu_{\mathscr{A}_{2}}\right)\left(u_{i}, v_{j}\right) \\
& =\sum_{u_{i} u_{k} \in E_{1}, v_{j} \neq v_{l}} \mu_{\mathscr{B}_{1}}\left(u_{i} u_{k}\right)+\mu_{\mathscr{A}_{1}}\left(u_{i}\right) \vee \mu_{\mathscr{A}_{2}}\left(v_{j}\right) \\
& =\left\{\begin{array}{cl}
\left(d_{\mu}\right) \mathscr{G}_{1}\left(u_{i}\right)+\mu_{\mathscr{A}_{1}}\left(u_{i}\right) & \text { if }\left|V_{2}\right|>1, \\
\mu_{\mathscr{A}_{1}}\left(u_{i}\right) & \text { if }\left|V_{2}\right|=1,
\end{array}\right. \\
& =\left\{\begin{aligned}
\left(t d_{\mu}\right) \mathscr{G}_{1}\left(u_{i}\right), & \text { if }\left|V_{2}\right|>1, \\
\mu_{\mathscr{A}}\left(u_{i}\right), & \text { if }\left|V_{2}\right|=1 .
\end{aligned}\right. \\
& \left(t d_{v}\right)_{\mathscr{G}_{1}} \mathscr{G}_{2}\left(u_{i}, v_{j}\right) \\
& =\sum_{\left(u_{i}, v_{j}\right)\left(u_{k}, v_{l}\right) \in E_{1} \times E_{2}}\left(v_{\mathscr{B}_{1}} \cdot v_{\mathscr{B}_{1}}\right)\left(\left(u_{i}, v_{j}\right)\left(u_{k}, v_{l}\right)\right) \\
& +\left(v_{\mathscr{A}_{1}} \cdot v_{\mathscr{A}_{1}}\right)\left(u_{i}, v_{j}\right) \\
& =\sum_{u_{i} u_{k} \in E_{1}, v_{j} \neq v_{l}} v_{\mathscr{B}_{1}}\left(u_{i} u_{k}\right) \\
& +v_{\mathscr{A}_{1}}\left(u_{i}\right) \wedge v_{\mathscr{A}_{2}}\left(v_{j}\right) \\
& =\left\{\begin{array}{cl}
\left(d_{v}\right) \mathscr{G}_{1}\left(u_{i}\right)+v_{\mathscr{A}_{1}}\left(u_{i}\right) & \text { if }\left|V_{2}\right|>1, \\
v_{\mathscr{A}_{1}}\left(u_{i}\right) & \text { if }\left|V_{2}\right|=1,
\end{array}\right. \\
& =\left\{\begin{array}{cl}
\left(t d_{v}\right) \mathscr{G}_{1}\left(u_{i}\right) & \text { if }\left|V_{2}\right|>1, \\
v_{\mathscr{A}_{1}}\left(u_{i}\right) & \text { if }\left|V_{2}\right|=1 .
\end{array}\right.
\end{aligned}
$$

(a) $\mathscr{G}_{1}$
$(0.9,0.2)$
$(0.8,0.3)$

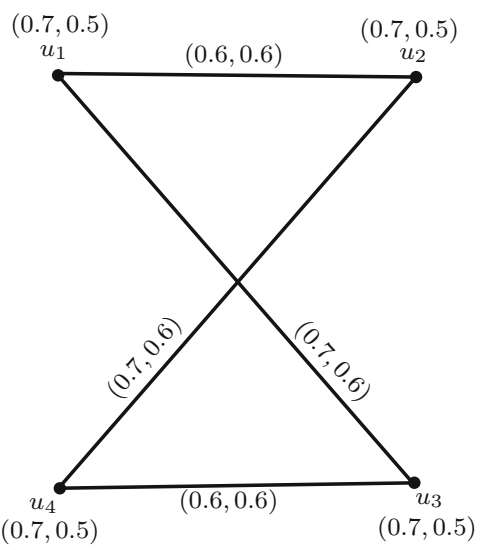

Fig. $26 \mathscr{G}=(\mathscr{A}, \mathscr{B})$

Definition 3.2 Let $\mathscr{G}=(\mathscr{A}, \mathscr{B})$ be a Pythagorean fuzzy graph on underlying crisp graph $G=(V, E)$. If

$$
\begin{aligned}
& t d_{\mu}(u)=\sum_{u, v \neq u \in V} \mu_{\mathscr{B}}(u v)+\mu(u)=k, \\
& t d_{v}(u)=\sum_{u, v \neq u \in V} v_{\mathscr{B}}(u v)+v(u)=l \quad \text { for all } \mathrm{u} \in \mathrm{V},
\end{aligned}
$$

then $\mathscr{G}$ is said to be totally regular Pythagorean fuzzy graph of total degree $(k, l)$ or $(k, l)$-totally regular PFG.

Example 3.2 Consider a graph $G=(V, E)$ where $V=$ $\left\{u_{1}, u_{2}, u_{3}, u_{4}\right\}$ and $E=\left\{u_{1} u_{2}, u_{1} u_{3}, u_{2} u_{4}, u_{3} u_{4}\right\}$. Let $\mathscr{A}$ and $\mathscr{B}$ be Pythagorean fuzzy vertex set and Pythagorean fuzzy edge set defined on $V$ and $V \times V$, respectively (Fig. 26).

$$
\begin{aligned}
\mathscr{A}= & \left\langle\left(\frac{u_{1}}{0.7}, \frac{u_{2}}{0.7}, \frac{u_{3}}{0.7}, \frac{u_{4}}{0.7}\right),\right. \\
& \left.\times\left(\frac{u_{1}}{0.5}, \frac{u_{2}}{0.5}, \frac{u_{3}}{0.5}, \frac{u_{4}}{0.5}\right)\right\rangle \text { and } \\
\mathscr{B}= & \left\langle\left(\frac{u_{1} u_{2}}{0.6}, \frac{u_{1} u_{3}}{0.7}, \frac{u_{2} u_{4}}{0.7}, \frac{u_{3} u_{4}}{0.6}\right),\right. \\
& \left.\times\left(\frac{u_{1} u_{2}}{0.6}, \frac{u_{1} u_{3}}{0.6}, \frac{u_{2} u_{4}}{0.6}, \frac{u_{3} u_{4}}{0.6}\right)\right\rangle .
\end{aligned}
$$


Since $t d_{\mu}\left(u_{i}\right)=1.8$ and $t d_{v}\left(u_{i}\right)=1.7$ for all $\mathrm{u}_{\mathrm{i}} \in V$ and $i=1,2,3,4$. Hence, $\mathscr{G}$ is a totally regular Pythagorean fuzzy graph of total degree $(1.8,1.7)$ or $(1.8,1.7)$-totally regular PFG.

Theorem 3.6 If $\mathscr{G}_{1}=\left(\mathscr{A}_{1}, \mathscr{B}_{1}\right)$ is a totally regular $P F G$ and $\mathscr{G}_{2}=\left(\mathscr{A}_{2}, \mathscr{B}_{2}\right)$ is a PFG such that $\mu_{\mathscr{A}_{1}} \geq \mu_{\mathscr{A}_{2}}, v_{\mathscr{A}_{1}} \leq v_{\mathscr{A}_{2}}$ and $\left|V_{2}\right|>1$, then the residue product is totally regular $P F G$.

Proof Let $\mathscr{G}_{1}=\left(\mathscr{A}_{1}, \mathscr{B}_{1}\right)$ be a $(k, l)$-totally regular PFG and $\mathscr{G}_{2}=\left(\mathscr{A}_{2}, \mathscr{B}_{2}\right)$ be a PFG such that $\mu_{\mathscr{A}_{1}} \geq \mu_{\mathscr{A}_{2}}, v_{\mathscr{A}_{1}} \leq$ $v_{\mathscr{A}_{2}}$ and $\left|V_{2}\right|>1$. Then $\left(t d_{\mu}\right)_{\mathscr{G}_{1}}\left(u_{i}\right)=k,\left(t d_{v}\right)_{\mathscr{G}_{1}}\left(u_{i}\right)=$ $l$, for all $u_{i}$ in $V_{1}$ and $\left(\mu_{\mathscr{A}_{1}} \cdot \mu_{\mathscr{A}_{2}}\right)\left(u_{1}, v_{1}\right)=\mu_{\mathscr{A}_{1}}\left(u_{1}\right)$, $\left(v_{\mathscr{A}_{1}} \cdot v_{\mathscr{A}_{2}}\right)\left(u_{1}, v_{1}\right)=v_{\mathscr{A}_{1}}\left(u_{1}\right)$, for all $\left(u_{1}, v_{1}\right)$ in $V_{1} \times V_{2}$. Now,

$$
\begin{aligned}
\left(t d_{\mu}\right) \mathscr{G}_{1} \cdot \mathscr{G}_{2}\left(u_{1}, v_{1}\right) & \\
& =\left(d_{\mu}\right) \mathscr{G}_{1} \cdot \mathscr{G}_{2}\left(u_{1}, v_{1}\right)+\left(\mu_{\mathscr{A}_{1}} \cdot \mu_{\mathscr{A}_{2}}\right)\left(u_{1}, v_{1}\right) \\
& =\left(d_{\mu}\right) \mathscr{G}_{1}\left(u_{1}\right)+\mu_{\mathscr{A}_{1}}\left(u_{1}\right) \\
& =\left(t d_{\mu}\right) \mathscr{G}_{1}\left(u_{1}\right) \\
& =k
\end{aligned}
$$

This is constant for all vertices in $V_{1} \times V_{2}$. Hence, $\mathscr{G}_{1} \cdot \mathscr{G}_{2}$ is a $(k, l)$-totally regular PFG.

\section{Applications to decision-making}

In this section, we apply the concept of PFGs to decisionmaking problems. Two decision-making problems concerning the 'evaluation of best company for investment' and 'alliance partner selection of a software company' are solved to illustrate the applicability of the proposed concept of PFGs in realistic scenario based on Pythagorean fuzzy preference relations (PFPRs) [31]. The algorithm for the alliance partner selection of a software company within the framework of PFPR is outlined in Algorithm 1.

\section{Evaluation of best company for investment}

To get the highest possible profit, assume that an investor wants to invest some money in an enterprise. Initially, he considers six possible alternatives: $x_{1}$ is a manufacturing company, $x_{2}$ is a fishing company, $x_{3}$ is a clothing company, $x_{4}$ is a feed company, $x_{5}$ is a seed company and $x_{6}$ is a building materials company. To prioritize these six possible

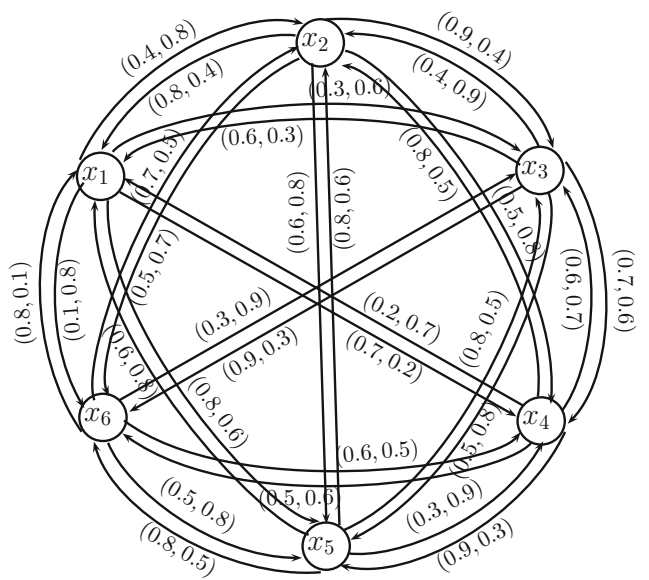

Fig. 27 Directed network of the Pythagorean fuzzy preference relation

Table 1 PFPR of the decision-maker

\begin{tabular}{lllllll}
\hline$R$ & $x_{1}$ & $x_{2}$ & $x_{3}$ & $x_{4}$ & $x_{5}$ & $x_{6}$ \\
\hline$x_{1}$ & $(0.5,0.5)$ & $(0.4,0.8)$ & $(0.3,0.6)$ & $(0.7,0.2)$ & $(0.8,0.6)$ & $(0.1,0.8)$ \\
$x_{2}$ & $(0.8,0.4)$ & $(0.5,0.5)$ & $(0.9,0.4)$ & $(0.5,0.8)$ & $(0.6,0.8)$ & $(0.5,0.7)$ \\
$x_{3}$ & $(0.6,0.3)$ & $(0.4,0.9)$ & $(0.5,0.5)$ & $(0.7,0.6)$ & $(0.5,0.8)$ & $(0.9,0.3)$ \\
$x_{4}$ & $(0.2,0.7)$ & $(0.8,0.5)$ & $(0.6,0.7)$ & $(0.5,0.5)$ & $(0.9,0.3)$ & $(0.5,0.6)$ \\
$x_{5}$ & $(0.6,0.8)$ & $(0.8,0.6)$ & $(0.8,0.5)$ & $(0.3,0.9)$ & $(0.5,0.5)$ & $(0.8,0.5)$ \\
$x_{6}$ & $(0.8,0.1)$ & $(0.7,0.5)$ & $(0.3,0.9)$ & $(0.6,0.5)$ & $(0.5,0.8)$ & $(0.5,0.5)$ \\
\hline
\end{tabular}

alternatives $\mathscr{X}=\left\{x_{1}, x_{1}, x_{3}, x_{4}, x_{5}, x_{6}\right\}$, a decision-maker is invited to assess them. The decision-maker compares each pair of these alternatives and provides the judgements by a directed network of the PFPR whose vertices represent these alternatives as shown in Fig. 27 and corresponding PFPRs [31] are shown in Table 1:

Using Pythagorean fuzzy averaging (PFA) operator [5],

$$
\begin{aligned}
p_{i} & =\operatorname{PFA}\left(p_{i 1}, p_{i 2}, \ldots, p_{i n}\right) \\
& =\left(\sqrt{1-\left(\prod_{j=1}^{n}\left(1-\mu_{i j}^{2}\right)\right)^{1 / n}},\left(\prod_{j=1}^{n} v_{i j}\right)^{1 / n}\right), \quad i=1,2,3, \ldots, n,
\end{aligned}
$$

we aggregate all $p_{i j}, j=1,2, \ldots, 6$ corresponding to the company $x_{i}$, and then get the complex Pythagorean fuzzy number $p_{i}$ of the company $x_{i}$, over all the other companies:

$$
\begin{aligned}
& p_{1}=(0.5604,0.5334), p_{2}=(0.6970,0.5742) \\
& p_{3}=(0.6669,0.5185) \\
& p_{4}=(0.6814,0.5295), p_{5}=(0.6909,0.6148) \\
& p_{6}=(0.6116,0.4561)
\end{aligned}
$$


Table 2 PFPR of the expert from the business process outsourcing department

\begin{tabular}{llllll}
\hline$R_{1}$ & $x_{1}$ & $x_{2}$ & $x_{3}$ & $x_{4}$ & $x_{5}$ \\
\hline$x_{1}$ & $(0.5,0.5)$ & $(0.5,0.7)$ & $(0.8,0.4)$ & $(0.7,0.6)$ & $(0.3,0.6)$ \\
$x_{2}$ & $(0.7,0.5)$ & $(0.5,0.5)$ & $(0.2,0.9)$ & $(0.7,0.5)$ & $(0.8,0.3)$ \\
$x_{3}$ & $(0.4,0.8)$ & $(0.9,0.2)$ & $(0.5,0.5)$ & $(0.2,0.6)$ & $(0.9,0.2)$ \\
$x_{4}$ & $(0.6,0.7)$ & $(0.5,0.7)$ & $(0.6,0.2)$ & $(0.5,0.5)$ & $(0.3,0.7)$ \\
$x_{5}$ & $(0.6,0.3)$ & $(0.3,0.8)$ & $(0.2,0.9)$ & $(0.7,0.3)$ & $(0.5,0.5)$ \\
\hline
\end{tabular}

Utilize the score function $s\left(p_{i}\right)=\mu_{i}^{2}-v_{i}^{2}[6]$ in order to calculate the scores of $p_{i}, i=1,2, \ldots, 6$ :

$s\left(p_{1}\right)=0.0295, s\left(p_{2}\right)=0.1562, s\left(p_{3}\right)=0.1759$,

$s\left(p_{4}\right)=0.1839, s\left(p_{5}\right)=0.0994, s\left(p_{6}\right)=0.1660$.

According to $s\left(p_{i}\right), i=1,2, \ldots, 6$, we get the ranking of the partners $x_{i}, i=1,2, \ldots, 6$ as:

$x_{4} \succ x_{3} \succ x_{6} \succ x_{2} \succ x_{5} \succ x_{1}$.

Therefore, the best company is $x_{4}$ to get the highest possible profit.

\section{Alliance partner selection of a software company}

Eastsoft is one of the top five software companies in China [36]. It offers a rich portfolio of businesses, including product engineering solutions, industry solutions, and related software products and platform and services. It is dedicated to becoming a globally leading IT solutions and services provider through continuous improvement of organization and process, competence development of leadership and employees, and alliance and open innovation. To improve the operation and competitiveness capability in the global market, Eastsoft plans to establish a strategic alliance with a transnational corporation. After numerous consultations, five transnational corporations (alternatives) would like to establish a strategic alliance with Eastsoft; they are HP $x_{1}$, PHILIPS $x_{2}$, EMC $x_{3}$, SAP $x_{4}$ and LK $x_{5}$. To select the desirable strategic alliance partner, a committee comprising three decision-makers $m_{k}(k=1,2,3)$ (whose weight vector is $w=(0.3,0.5,0.7))$ is founded, come from the business process outsourcing department, the operation management department and the engineering management department of Eastsoft, respectively. Based on their experiences, the decision-makers compare each pair of alternatives and give individual judgments using the following PFPRs $R_{k}=\left(p_{i j}^{(k)}\right)_{5 \times 5}(k=1,2,3)$ :

The Pythagorean fuzzy digraphs $\mathcal{D}_{k}$ corresponding to PFPRs $R_{k}(k=1,2,3)$ given in Tables 2,3 and 4, are shown in Fig. 28.
Table 3 PFPR of the expert from the operation management department

\begin{tabular}{llllll}
\hline$R_{2}$ & $x_{1}$ & $x_{2}$ & $x_{3}$ & $x_{4}$ & $x_{5}$ \\
\hline$x_{1}$ & $(0.5,0.5)$ & $(0.9,0.3)$ & $(0.7,0.2)$ & $(0.3,0.8)$ & $(0.5,0.8)$ \\
$x_{2}$ & $(0.3,0.9)$ & $(0.5,0.5)$ & $(0.6,0.7)$ & $(0.1,0.5)$ & $(0.8,0.6)$ \\
$x_{3}$ & $(0.2,0.7)$ & $(0.7,0.6)$ & $(0.5,0.5)$ & $(0.7,0.5)$ & $(0.3,0.7)$ \\
$x_{4}$ & $(0.8,0.3)$ & $(0.5,0.1)$ & $(0.5,0.7)$ & $(0.5,0.5)$ & $(0.8,0.3)$ \\
$x_{5}$ & $(0.8,0.5)$ & $(0.6,0.8)$ & $(0.7,0.3)$ & $(0.3,0.8)$ & $(0.5,0.5)$ \\
\hline
\end{tabular}

Table 4 PFPR of the expert from the engineering management department

\begin{tabular}{llllll}
\hline$R_{3}$ & $x_{1}$ & $x_{2}$ & $x_{3}$ & $x_{4}$ & $x_{5}$ \\
\hline$x_{1}$ & $(0.5,0.5)$ & $(0.7,0.6)$ & $(0.5,0.8)$ & $(0.3,0.9)$ & $(0.7,0.6)$ \\
$x_{2}$ & $(0.6,0.7)$ & $(0.5,0.5)$ & $(0.8,0.6)$ & $(0.1,0.7)$ & $(0.3,0.8)$ \\
$x_{3}$ & $(0.8,0.5)$ & $(0.6,0.8)$ & $(0.5,0.5)$ & $(0.4,0.8)$ & $(0.5,0.7)$ \\
$x_{4}$ & $(0.9,0.3)$ & $(0.7,0.1)$ & $(0.8,0.4)$ & $(0.5,0.5)$ & $(0.8,0.4)$ \\
$x_{5}$ & $(0.6,0.7)$ & $(0.8,0.3)$ & $(0.7,0.5)$ & $(0.4,0.8)$ & $(0.5,0.5)$ \\
\hline
\end{tabular}

Compute the averaged pythagorean fuzzy element (PFE) $p_{i}^{(k)}$ of the alternative $x_{i}$ over all the other alternatives for the experts $m_{k}(k=1,2,3)$ by the Pythagorean fuzzy averaging (PFA) operator:

$$
\begin{aligned}
p_{i}^{(k)} & =P F A\left(p_{i 1}^{(k)}, p_{i 2}^{(k)}, \ldots, p_{i n}^{(k)}\right) \\
& =\left(\sqrt{1-\left(\prod_{j=1}^{n}\left(1-\mu_{i j}^{2}\right)\right)^{1 / n}},\left(\prod_{j=1}^{n} v_{i j}\right)^{1 / n}\right), \quad i=1,2,3, \ldots, n .
\end{aligned}
$$

The aggregation results of the experts $m_{k}(k=1,2,3)$ are as follows:

$$
\begin{aligned}
m_{1}: p_{1}^{(1)} & =(0.6139,0.5502), p_{2}^{(1)}=(0.6457,0.5078), \\
p_{3}^{(1)} & =(0.7312,0.3949), p_{4}^{(1)}=(0.5180,0.5094), \\
p_{5}^{(1)} & =(0.5152,0.5036) ; \\
m_{2}: p_{1}^{(2)} & =(0.6720,0.4536), p_{2}^{(2)}=(0.5574,0.6239), \\
p_{3}^{(2)} & =(0.5459,0.5933), p_{4}^{(2)}=(0.6639,0.3160), \\
p_{5}^{(2)} & =(0.6295,0.5448) ; \\
m_{3}: p_{1}^{(3)} & =(0.5761,0.6645), p_{2}^{(3)}=(0.5574,0.6518), \\
p_{3}^{(3)} & =(0.5985,0.6454), p_{4}^{(3)}=(0.7789,0.2993), \\
p_{5}^{(3)} & =(0.6371,0.5305) .
\end{aligned}
$$

Compute a collective PFE $p_{i}(i=1,2, \ldots, n)$ of the alternative $x_{i}$ over all the other alternatives using the Pythagorean fuzzy weighted averaging (PFWA) operator [5]:

$$
\begin{aligned}
& p_{i}=\operatorname{PFWA}\left(p_{i}^{(1)}, p_{i}^{(2)}, \ldots, p_{i}^{(s)}\right) \\
& =\left(\sqrt{1-\prod_{k=1}^{s}\left(1-\left(\mu_{k}^{2}\right)\right)^{w_{k}}}, \prod_{k=1}^{s}\left(v_{k}\right)^{w_{k}}\right) \text {. } \\
& \text { مدينة الملك عبدالعزيز }
\end{aligned}
$$

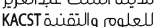


Fig. 28 Pythagorean fuzzy digraphs

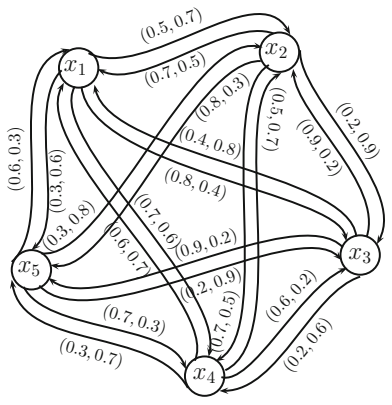

$\mathcal{D}_{1}$

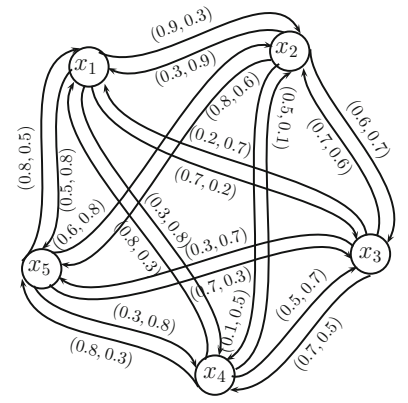

$\mathcal{D}_{2}$

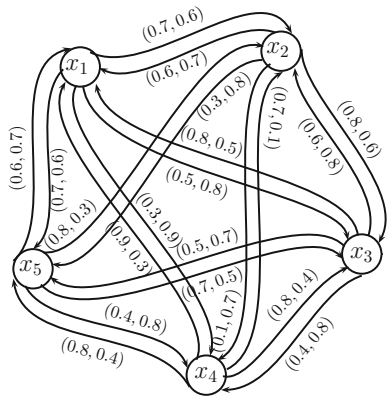

$\mathcal{D}_{3}$

Table 5 The decision results of the alternatives using the different methods

\begin{tabular}{lrrrrrr}
\hline Methods & Score of alternatives & Ranking of alternatives \\
\hline Zhao et al. [40] & -0.6800 & -2.1800 & -0.9400 & 3.8200 & -0.0200 & $A_{4} \succ A_{5} \succ A_{1} \succ A_{3} \succ A_{2}$ \\
Our proposed method & 0.3367 & 0.2276 & 0.3276 & 0.6067 & 0.3595 & $A_{4} \succ A_{5} \succ A_{1} \succ A_{3} \succ A_{2}$ \\
\hline
\end{tabular}

That is,

$p_{1}=(0.7180,0.4229), p_{2}=(0.6751,0.4777)$,

$p_{3}=(0.7153,0.4290)$,

$p_{4}=(0.8035,0.1973), p_{5}=(0.7128,0.3855)$.

Compute the score functions $s\left(p_{i}\right)=\mu_{i}^{2}-v_{i}^{2}[6]$ of $p_{i}(i=$ $1,2,3,4,5)$, and rank all the alternatives $x_{i}(i=1,2,3,4,5)$ according to the values of $s\left(p_{i}\right)(i=1,2,3,4,5)$.

$s\left(p_{1}\right)=0.3367, s\left(p_{2}\right)=0.2276, s\left(p_{3}\right)=0.3276$,

$s\left(p_{4}\right)=0.6067, s\left(p_{5}\right)=0.3595$.

Then $x_{4} \succ x_{5} \succ x_{1} \succ x_{3} \succ x_{2}$. Thus, the optimal choice is SAP $x_{4}$.

Comparative Analysis: We individually compute and compare the decision results using the method of Ref. [40] and our proposed method. They are shown in Table 5. From Table 5, the decision results of the method of Ref. [40] are consistent with our proposed method. It implies that our proposed method considers all the Pythagorean fuzzy evaluation information.

\section{Conclusions and further work}

Graph theory has vast range of applications in solving various networking problems encountered in different fields such as signal processing, transportation and error-correcting codes. For modeling the obscurity and uncertainties in practical decision-making and graphical networking problems, Pythagorean fuzzy graphs (PFGs) have better ability due to the increment of spaces in membership and non-membership
Algorithm 1 The algorithm for selection of alliance partner of a software company.

INPUT: A discrete set of alternatives $\mathscr{X}=\left\{x_{1}, x_{2}, \ldots, x_{n}\right\}$, a set of experts $e=\left\{m_{1}, m_{2}, \ldots, m_{s}\right\}$ with a weight vector $w=$ $\left\{w_{1}, w_{1}, \ldots, w_{s}\right\}$ and construction of PFPR $R_{k}=\left(p_{i j}^{(k)}\right)_{n \times n}$ for each expert.

OUTPUT: The selection of optimal alternative.

1. begin

2. Aggregate all $p_{i j}^{(k)}(j=1,2, \ldots, n)$ corresponding to the alternative $x_{i}$ and get the PFE $p_{i}^{(k)}$ of the alternative $x_{i}$ over all the other alternatives for the expert $m_{k}$ by using the PFA operator.

$$
\begin{aligned}
p_{i}^{(k)}= & P F A\left(p_{i 1}^{(k)}, p_{i 2}^{(k)}, \ldots, p_{i n}^{(k)}\right) \\
= & \left(\sqrt{1-\left(\prod_{j=1}^{n}\left(1-\mu_{i j}^{2}\right)\right)^{1 / n}},\left(\prod_{j=1}^{n} v_{i j}\right)^{1 / n}\right), \\
& i=1,2,3, \ldots, n .
\end{aligned}
$$

3. Aggregate all $p_{i}^{(k)}(k=1,2, \ldots, s)$ into a collective PFE $p_{i}$ for the alternative $x_{i}$ using the PFWA operator.

$$
\begin{aligned}
p_{i} & =\operatorname{PFWA}\left(p_{i}^{(1)}, p_{i}^{(2)}, \ldots, p_{i}^{(s)}\right) \\
& =\left(\sqrt{1-\prod_{k=1}^{s}\left(1-\left(\mu_{k}^{2}\right)\right)^{w_{k}}, \prod_{k=1}^{s}\left(v_{k}\right)^{w_{k}}}\right) .
\end{aligned}
$$

4. Compute the score functions $s\left(p_{i}\right)=\mu_{i}^{2}-v_{i}^{2}$ of $p_{i}(i=$ $1,2, \ldots, n)$.

5. Rank all the alternatives $x_{i}(i=1,2, \ldots, n)$ according to $s\left(p_{i}\right)(i=$ $1,2, \ldots, n)$.

6. Output the optimal alternative.

7. end

grades. This paper has introduced two new graph products specifically, maximal product and residue product, for uniting two Pythagorean fuzzy graphs. Using these products, different types of structural models can be combined to 
produce a better one. They may be useful for the configuration processing of space structures. Further, the paper has explored some crucial properties like strongness, connectedness, completeness and regularity. A special focus on the regularity has been given as it can be applied widely in designing reliable communication and computer networks. Applications of PFGs in decision-making concerning evaluation of best company for investment and alliance partner selection of a software company have been presented.

Further work We are working to extend our study to : (1) Pythagorean fuzzy soft graphs; (2) Rough Pythagorean fuzzy graphs; (3) Simplified interval-valued Pythagorean fuzzy graphs and; (4) Hesitant Pythagorean fuzzy graphs

Acknowledgements The authors are highly thankful to Associate Editor anonymous referees for their valuable comments and suggestions for improving the paper.

\section{Compliance with ethical standards}

Conflict of interest The authors declare that they have no conflict of interest regarding the publication of the research article.

Open Access This article is distributed under the terms of the Creative Commons Attribution 4.0 International License (http://creativecomm ons.org/licenses/by/4.0/), which permits unrestricted use, distribution, and reproduction in any medium, provided you give appropriate credit to the original author(s) and the source, provide a link to the Creative Commons license, and indicate if changes were made.

\section{References}

1. Zadeh LA (1965) Fuzzy sets. Inf Control 8(3):338-353

2. Atanassov KT (1983) Intuitionistic fuzzy sets, VII ITKR's Session, Sofia, Deposed in Central Sci. - Techn. Library of Bulg. Acad. of Sci., 1697/84 (in Bulgarian)

3. Yager RR (2013) Pythagorean fuzzy subsets. In: Proceedings of the Joint IFSA World Congress and NAFIPS Annual Meeting, Edmonton, Canada, pp 57-61

4. Yager RR, Abbasov AM (2013) Pythagorean membership grades, complex numbers, and decision making. Int J Intell Syst 28(5):436452

5. Yager RR (2014) Pythagorean membership grades in multi-criteria decision making. IEEE Trans Fuzzy Syst 22(4):958-965

6. Zhang X, Xu Z (2014) Extension of TOPSIS to multiple criteria decision making with Pythagorean fuzzy sets. Int J Intell Syst 29(12): 1061-1078

7. Peng X, Yang Y (2015) Some results for Pythagorean fuzzy sets. Int J Intell Syst 30(11):1133-1160

8. Rangasamy P, Palaniappan N (2003) Some operations on intuitionistic fuzzy sets of second type. Notes Intuit Fuzzy Sets 10(2):1-19

9. Ren P, Xu Z, Gou X (2016) Pythagorean fuzzy TODIM approach to multi-criteria decision making. Appl Soft Comput 42:246-259

10. Peng X, Selvachandran G (2017) Pythagorean fuzzy set: state of the art and future directions. Artif Intell Rev. https://doi.org/10. 1007/s10462-017-9596-9

11. Garg H (2018) A new exponential operational laws and their aggregation operators of interval-valued Pythagorean fuzzy information. Inte J Intell Syst 33(3):653-683
12. Garg H (2018) Some methods for strategic decision-making problems with immediate probabilities in Pythagorean fuzzy environment. Int J Intell Syst 33(4):687-712

13. Garg H (2018) Linguistic Pythagorean fuzzy sets and its applications in multi attribute decision making process. Int J Intell Syst 33(6): 1234-1263

14. Garg H (2018) Hesitant Pythagorean fuzzy sets and their aggregation operators in multiple-attribute decision-making. Int J Uncert Quantif 8(3):267-289

15. Garg H (2016) A novel correlation coefficients between Pythagorean fuzzy sets and its applications to decision-making processes. Int J Intell Syst 31(12):1234-1253

16. Garg H (2018) New Logarithmic operational laws and their aggregation operators for Pythagorean fuzzy set and their applications. Int J Intell Syst. https://doi.org/10.1002/int.22043.

17. Garg H (2018) Generalized Pythagorean fuzzy geometric interactive aggregation operators using Einstein operations and their application to decision making. J Exp Theor Artif Intell. https:// doi.org/10.1080/0952813X.2018.1467497.

18. Garg H (2017) Confidence levels based Pythagorean fuzzy aggregation operators and its application to decision-making process. Comput Math Organ Theory 23(4):546571

19. Garg H (2017) A new improved score function of an interval-valued pythagorean fuzzy set based TOPSIS method. Int J Uncert Quantif 7(5):463-474

20. Garg H (2018) A linear programming method based on an improved score function for interval-valued Pythagorean fuzzy numbers and its application to decision-making. Int J Uncert Fuzziness Knowl Based Syst 29(1):67-80

21. Yu SM, Zhang HY, Wang JG (2018) Hesitant Pythagorean fuzzy Maclaurin symmetric mean operators and its applications to multiattribute decision making process. Int J Intell Syst. https://doi.org/ 10.1002/int.22067.

22. Kaufmann A (1973) Introduction a la Theorie des Sour-ensembles Flous. Masson et Cie 1

23. Rosenfeld A (1975) In: Zadeh LA, Fu KS, Shimura M (eds) Fuzzy graphs, fuzzy sets and their applications. Academic Press, New York, pp 77-95

24. Nagoor Gani A, Radha K (2008) On regular fuzzy graphs. J Phys Sci 12:33-44

25. Mordeson JN, Peng CS (1994) Operations on fuzzy graphs. Inf Sci 79(3):159-170

26. Nirmala G, Vijaya M (2012) Fuzzy graphs on composition, tensor and normal products. Int J Sci Res Publ 2(6):1-7

27. Parvathi R, Karunambigai MG (2006) Intuitionistic fuzzy graphs. In: Intelligence computational, theory and applications. Springer, Berlin, pp 139-150

28. Akram M, Davvaz B (2012) Strong intuitionistic fuzzy graphs. Filomat 26(1):177-196

29. Sarwar M, Akram M (2016) An algorithm for computing certain metrics in intuitionistic fuzzy graphs. J Intell Fuzzy Syst 30(4):2405-2416

30. Akram M, Dudek WA (2013) Intuitionistic fuzzy hypergraphs with applications. Inf Sci 218:182-193

31. Naz S, Ashraf S, Akram M (2018) A novel approach to decisionmaking with Pythagorean fuzzy information. Mathematics 6(6):95

32. Akram M, Naz S (2018) Energy of Pythagorean fuzzy graphs with applications. Mathematics 6(8):136. https://doi.org/10.3390/ math6080136

33. Akram M, Dar JM, Farooq A (2018) Planar graphs under pythagorean fuzzy environment. Mathematics 6(12):278. https:// doi.org/10.3390/math6120278

34. Akram M, Habib A, Ilyas F, Dar JM (2018) Specific types of Pythagorean fyzzy graphs and application to decision-making. Math Comput Appl 23:42. https://doi.org/10.3390/mca23030042

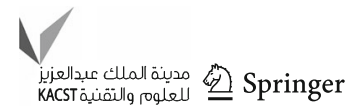


35. Dhavudh SS, Srinivasan R (2017) Properties of intuitionistic fuzzy graphs of second type, International. J Comput Appl Math 12(3):815-823

36. Fan ZP, Liu Y (2010) An approach to solve group decision-making problems with ordinal interval numbers. IEEE Trans Syst Man Cybern Part B (Cybernetics) 40(5)

37. Ghorai G, Pal M (2016) A study on $m$-polar fuzzy planar graphs. Int J Comput Sci Math 7(3):283-292

38. Ghorai G, Pal M, Some properties of $m$-polar fuzzy graphs. Pac Sci Rev A Nat Sci Eng. https://doi.org/10.1016/j.psra.2016.06.004
39. Mandal S, Sahoo S, Ghorai G, Pal M (2018) Application of strong arcs in m-polar fuzzy graphs. Neural Process Lett. https://doi.org/ 10.1007/s11063-018-9934-1

40. Wang R, Li YL (2018) A novel approach for group decision-making from intuitionistic fuzzy preference relations and intuitionistic multiplicative preference relations. Information 9(3):1-15

Publisher's Note Springer Nature remains neutral with regard to jurisdictional claims in published maps and institutional affiliations. 